\title{
ABSOLUTE CONTINUITY IN THE DUAL OF A BANACH ALGEBRA ${ }^{1}$
}

\author{
BY
}

\author{
STEPHEN JAY BERMAN
}

\begin{abstract}
If $A$ is a Banach algebra, $G$ is in the dual space $A^{*}$, and $I$ is a closed ideal in $A$, then let $\|G\|_{I^{*}}$ denote the norm of the restriction of $G$ to $I$. We define a relation $\ll$ in $A^{*}$ as follows: $G \ll L$ if for every $\varepsilon>0$ there exists a $\delta>0$ such that if $I$ is a closed ideal in $A$ and $\|L\|_{I^{*}}<\delta$ then $\|G\|_{I^{*}}<\varepsilon$. We explore this relation (which coincides with absolute continuity of measures when $A$ is the algebra of continuous functions on a compact space) and related concepts in the context of several Banach algebras, particularly the algebra $C^{1}[0,1]$ of differentiable functions and the algebra of continuous functions on the disc with holomorphic extensions to the interior. We also consider generalizations to noncommutative algebras and Banach modules.
\end{abstract}

Introduction. We wish to define a binary relation in the dual of a Banach algebra which will generalize the relation of absolute continuity for measures. While the general theorems that we are able to prove are trivial, the examples which follow seem to indicate that the concept is worth considering in the study of a particular Banach algebra.

1. Definitions. Let $X$ be a compact Hausdorff space and let $C(X)$ denote the space of real (or complex) valued continuous functions on $X$ with the supremum norm. If $\mu$ and $\nu$ are finite regular Borel measures on $X$, let $|\mu|$ and $|\nu|$ be the total variation measures of $\mu$ and $\nu$. Recall that $\nu$ is absolutely continuous with respect to $\mu$ if for every $\varepsilon>0$ there exists $\delta>0$ such that if $E$ is a Borel set in $X$ and $|\mu|(E)<\delta$ then $|\nu|(E)<\varepsilon$. The regularity of $\mu$ and $\nu$ implies that we have an equivalent condition if we restrict $E$ to be an open set. Now if $E$ is open, then $|\mu|(E)=\sup \left\{\left|\int f d \mu\right|: f \in C(X),\|f\|<1, f(x)=\right.$ 0 if $x \notin E\}$, that is, $|\mu|(E)$ is the norm of the linear functional $\mu$ restricted to the closed ideal $I=\{f \in C(X): f(x)=0$ if $x \notin E\}$. So we are led to the following definition:

Definition. Let $A$ be a commutative Banach algebra (over the real or

Received by the editors June 24, 1975.

AMS (MOS) subject classifications (1970). Primary 46H10, 46J10, 46J15, 46J20.

Key words and phrases. Ideals in Banach algebras, absolute continuity, Radon-Nikodym theorems.

'Part of this research was done under National Science Foundation Grant GP-30798X at the University of California, Berkeley. 
complex field) with dual $A^{*}$. If $L \in A^{*}$ and $I$ is a closed subspace of $A$, let $\|L\|_{I^{*}}$ denote the norm of the restriction of $L$ to $I$.

1. We say that $G \in A^{*}$ is absolutely continuous with respect to $L \in A^{*}$ (written $G \ll L$ ) if for every $\varepsilon>0$ there exists $\delta>0$ such that if $I$ is a closed ideal in $A$ and $\|L\|_{I^{*}}<\delta$ then $\|G\|_{I^{*}}<\varepsilon$.

2. We say that $G$ is boundedly absolutely continuous with respect to $L$ (written $G<<<L$ ) if there exists a constant $K$ such that $\|G\|_{I^{*}}<K\|L\|_{I^{*}}$ for every closed ideal $I$ in $A$.

3. We say that a closed subspace $B$ of $A^{*}$ is a band if $G \ll L$ and $L \in B$ imply $G \in B$.

Recall the following definitions in Arens [1], [2]:

4. If $f \in A$ and $L \in A^{*}$, then $f L$ is the linear functional on $A$ defined by $(f L)(g)=L(f g)$ for all $g \in A$. Clearly $f L \in A^{*}$ and $\|f L\|<\|f\|\|L\|$.

5. If $\Phi \in A^{* *}$ and $L \in A^{*}$, then $\Phi L$ is the linear functional on $A$ defined by $(\Phi L)(f)=\Phi(f L)$ for all $f \in A$. Clearly $\Phi L \in A^{*}$ and $\|\Phi L\|<\|\Phi\|\|L\|$.

6. If $\Phi, \Psi \in A^{* *}$, then $\Phi \Psi$ is the linear functional on $A^{*}$ defined by $(\Phi \Psi)(L)=\Phi(\Psi L)$. Clearly $\Phi \Psi \in A^{* *}$ and $\|\Phi \Psi\|<\|\Phi\|\|\Psi\|$. With this operation, $A^{* *}$ is a Banach algebra.

Proposition 1. 1. If $I$ is a closed ideal in $A, f \in I$, and $L \in A^{*}$, then $\|f L\| \leqslant\|L\|_{I^{*}}\|f\|$.

2. If $\Phi \in A^{* *}$ and $L \in A^{*}$, then $\|\Phi L\|_{I^{*}} \leqslant\|\Phi\|\|L\|_{I^{*}}$ for any closed ideal $I$ in $A$. In particular, $\Phi L<<<L$.

3. If $L \in A^{*}$, then $\left\{G \in A^{*}: G \ll L\right\}$ is a band, called the principal band generated by $L$.

Proof. 1. If $g \in A$ then $f g \in I$ and $|(f L)(g)|=|L(f g)|<\|L\|_{I^{*}}\|f g\|<$ $\|L\|_{I^{*}}\|f\|\|g\|$, so that $\|f L\|<\|L\|_{I^{*}}\|f\|$.

2. If $f \in I$, then $|(\Phi L)(f)|=|\Phi(f L)|<\|\Phi\|\|f L\|<\|\Phi\|\|f\|\|L\|_{I^{*} \text {, so }}$ that $\|\Phi L\|_{I^{*}}<\|\Phi\|\|L\|_{I^{* *}}$

3. The indicated set is clearly a subspace of $A^{*}$. To show it is closed, suppose $G_{n} \rightarrow G$ and $G_{n} \ll L$. Given $\varepsilon>0$, find $n$ so that $\left\|G_{n}-G\right\|<\varepsilon / 2$ and then find $\delta>0$ so that $\left\|G_{n}\right\|_{I^{*}}<\varepsilon / 2$ whenever $\|L\|_{I^{*}}<\delta$. Then $\|L\|_{I^{*}}$ $<\delta$ implies $\|G\|_{I^{*}}<\left\|G-G_{n}\right\|_{I^{*}}+\left\|G_{n}\right\|_{I^{*}}<\varepsilon / 2+\varepsilon / 2=\varepsilon$.

Let us consider again the algebra $C(X), X$ compact Hausdorff. The Radon-Nikodym Theorem states that if $\nu \ll \mu$ then $d \nu=F d \mu$ where $F$ is $\mu$-integrable. Since $C(X)$ is dense in the $\mu$-integrable functions, an equivalent statement of the Radon-Nikodym theorem is that $\nu \ll \mu$ if and only if $\nu$ is in the norm closure of $\{f \mu: f \in C(X)\}$.

For any Banach algebra $A$ and any $L \in A^{*}$, we have

$$
\{f L: f \in A\} \subset\left\{\Phi L: \Phi \in A^{* *}\right\} \subset\{G: G<<<L\} \subset\{G: G \ll L\}
$$


and we will be interested in finding which of these sets (or their closures) are equal.

Proposition 2. If Arens multiplication in $A^{* *}$ is commutative, that is, if $\Phi \Psi=\Psi \Phi$ for all $\Phi, \Psi \in A^{* *}$, then for any $L \in A$, the closures of $\{f L$ : $f \in A\}$ and $\left\{\Phi L: \Phi \in A^{* *}\right\}$ are equal.

Proor. If the closures are not equal, then by the Hahn-Banach theorem we can find a nonzero $\Psi \in A^{* *}$ such that $\Psi(f L)=0$ for all $f \in A$ but $\Psi(\Phi L) \neq$ 0 for some $\Phi \in A^{* *}$. But then $(\Psi L)(f)=\Psi(f L)=0$ for all $f$, so $\Psi L=0$ and $\Psi(\Phi L)=\Phi(\Psi L)=0$, a contradiction.

If $A$ is a uniform algebra (that is, a closed subalgebra of $C(X)$ for some compact Hausdorff space $X$ ) then $A^{* *}$ is commutative, (Civin and Yood [3, p. 869]) so that Proposition 2 applies to $A$.

\section{Examples.}

EXAMPLE 1. Let $A$ be any Banach space and define multiplication in $A$ by $f g=0$ for all $f, g \in A$. Then every subspace of $A$ is an ideal. So if $L \in A^{*}$, then the kernel of $L$ is an ideal, and $G \ll L$ and $G<<<L$ both imply that $G$ is zero on the kernel of $L$, so that $G$ is a scalar multiple of $L$. Hence $\{G$ : $G \ll L\}=\{G: G<<<L\}=\{\lambda L: \lambda$ any scalar $\}$. However, $\{f L: f \in A\}=$ $\left\{\Phi L: \Phi \in A^{* *}\right\}=\{0\}$.

EXAMPLE 2. Let $A$ be a commutative Banach algebra and suppose that $L$ is a nonzero multiplicative linear functional on $A$. Then the kernel of $L$ is an ideal, so we find (as in Example 1) that $\{G: G \ll L\}=\{G: G<<<L\}=$ $\{\lambda L: \lambda$ scalar $\}$. Furthermore, $f L=L(f) L$ and $\Phi L=\Phi(L) L$, so that $\{f L$ : $f \in A\}=\left\{\Phi L: \Phi \in A^{* *}\right\}=\{\lambda L: \lambda$ scalar $\}$.

EXAMPLE 3. Let $A$ be a commutative Banach algebra with identity $e$. Let $M$ be a nonzero multiplicative linear functional on $A$ and let $L$ be a nonzero bounded point derivation of $M$, that is, $L \in A^{*}$ and $L(f g)=L(f) M(g)+$ $M(f) L(g)$ for all $f, g \in A$. Clearly $f L=L(f) M+M(f) L$ if $f \in A$ and $\Phi L=\Phi(L) M+\Phi(M) L$ if $\Phi \in A^{* *}$, so that $\{f L: f \in A\}=\{\Phi L: \Phi \in$ $\left.A^{* *}\right\}=\left\{\lambda_{1} M+\lambda_{2} L: \lambda_{1}, \lambda_{2}\right.$ scalar $\}$. We claim that if $G \ll L$, then $G=\lambda_{1} M$ $+\lambda_{2} L$ for some scalars $\lambda_{1}$ and $\lambda_{2}$. To show this, let $J$ be the kernel of $M$. Then $J$ is a maximal ideal in $A$. Let $I=\{f \in J: L(f)=0\}$. Now $I$ is an ideal in $A$, for if $f \in I$ and $g \in A$, then we can write $g$ in the form $g=j+\lambda$, where $j \in J$ and $\lambda$ is a scalar, and since $L(f j)=L(f) M(j)+M(f) L(j)=0$, we find that $f j \in I$ and $f g=f j+\lambda f \in I$. Since $L=0$ on $I$ and $G \ll L$, we see that $G=0$ on $I$. Now $I$ is the kernel of the restriction of $L$ to $J$, so that $G$ must be a scalar multiple of $L$ on $J$. That is, there exists a scalar $\lambda_{2}$ such that $G=\lambda_{2} L$ on $J$. Hence $G=G(e) M+\lambda_{2} L$ on $J$, and since this equality also holds for the identity $e$, we have $G=G(e) M+\lambda_{2} L$ on all of $A$, as wanted.

EXAMPLE 4. Let $A=P_{n}$ be the algebra of polynomials over the real (or 
complex) field in a single indeterminate $x$ such that $x^{n+1}=0$. As a vector space $P_{n}$ is just $\mathbf{R}^{n+1}$ and we give it the norm $\left\|\sum_{j=0}^{n} a_{j} x^{j}\right\|=\sum_{j=0}^{n}\left|a_{j}\right|$. The dual of $P_{n}$ is $\mathbf{R}^{n+1}$, every $L \in P_{n}^{*}$ having the form $L\left(\Sigma_{j=0}^{n} a_{j} x^{j}\right)=\sum_{j=0}^{n} a_{j} \mu_{j}$, $\left(\mu_{0}, \ldots, \mu_{n}\right) \in \mathbf{R}^{n+1}$; furthermore, $\|L\|=\max \left\{\left|\mu_{j}\right|: j=0, \ldots, n\right\}$. For each $m$ such that $0<m<n$, the set $I_{m}=\left\{\sum_{j=0}^{n} a_{j} x^{j}: a_{j}=0\right.$ if $\left.j<m\right\}$ is a closed ideal in $P_{n}$, and these are readily seen to be the only ideals. Note that $\|L\|_{I_{m}^{*}}=\max \left\{\left|\mu_{j}\right|: j>m\right\}$ if $m<n$.

The relations $\ll$ and $<<<$ in $P_{n}^{*}$ can be described as follows. Suppose that $L\left(\sum_{j=0}^{n} a_{j} x^{j}\right)=\sum_{j=0}^{n} a_{j} \mu_{j}$, and $G\left(\sum_{j=0}^{n} a_{j} x^{j}\right)=\sum_{j=0}^{n} a_{j} \nu_{j}$ and $m=\max \{j$ : $\left.\mu_{j} \neq 0\right\}$. Then the following are equivalent: (i) $G=g L$ for some $g \in P_{n}$; (ii) $G<<<L$; (iii) $G \ll L$; and (iv) $\nu_{j}=0$ if $j>m$. Clearly (i) $\Rightarrow$ (ii) $\Rightarrow$ (iii). If (iii) holds, then since $\|L\|_{I_{m}^{*}}=0$, it follows that $\|G\|_{I_{m}^{*}}=0$, hence $\nu_{j}=0$ if $j>m$, proving (iv). Finally, if (iv) holds then we need to find $g$ so that $g L=G$. Note that if $g=\sum_{j=0}^{n} b_{j} x^{j}$ and $f=\sum_{j=0}^{n} a_{j} x^{j}$, then

$$
\begin{aligned}
(g L)(f) & =L(f g)=L\left[\sum_{i=0}^{n}\left(\sum_{j=0}^{i} a_{j} b_{i-j}\right) x^{i}\right] \\
& =\sum_{i=0}^{n} \mu_{i} \sum_{j=0}^{i} a_{j} b_{i-j}=\sum_{j=0}^{n}\left(\sum_{i=j}^{n} \mu_{i} b_{i-j}\right) a_{j},
\end{aligned}
$$

so that $g L=G$ if and only if

$$
\sum_{i=j}^{n} \mu_{i} b_{i-j}=\nu_{j} \quad(j=0,1, \ldots, n) .
$$

For $j>m$, both sides of this equation are 0 . For $j=m, m-1, m-$ $2, \ldots, 1,0$ the equations can be solved successively for $b_{0}, b_{1}, \ldots, b_{m}$ since $\mu_{m} \neq 0$. Then $b_{m+1}, \ldots, b_{n}$ can be given arbitrary values.

In particular note that if $G \ll L$ then $G$ is in the closure of $\left\{g L: g \in P_{n}\right\}$. This property is not shared by the following algebra.

Let $P_{n}^{0}$ be the algebra $I_{1}$, that is, the polynomials with constant term equal to 0 . The ideals in $P_{n}^{0}$ are $I_{m}, m=1, \ldots, n$, as before. If $L\left(\sum_{j=1}^{n} a_{j} x^{j}\right)=$ $\sum_{j=1}^{n} a_{j} \mu_{j}$ and $G\left(\sum_{j=1}^{n} a_{j} x^{j}\right)=\sum_{j=1}^{n} a_{j} \nu_{j}$, and if $m=\max \left\{j: \mu_{j} \neq 0\right\}$, then

(a) each of the conditions $G \ll L$ and $G<<<L$ holds if and only if $\nu_{j}=0$ for $j>m$, and

(b) $G=g L$ for some $g \in P_{n}^{0}$ if and only if $\nu_{j}=0$ for $j>m$.

Statement (a) is easily proven as before. As for (b), if $G=g L$ and $j>m$, let $f_{j}=x^{j}$. Then $v_{j}=G\left(f_{j}\right)=L\left(g f_{j}\right)=0$. Conversely, if $\nu_{j}=0$ for $j>m$, we need to find $g$ so that $G=g L$. But if $g=\sum_{j=1}^{n} b_{j} x^{j}$, then as before $G=g L$ if and only if (*) holds for $j=1,2, \ldots, n-1$. Both sides are 0 for $j \geqslant m$, and for $j=m-1, m-2, \ldots, 2,1$, the equations can be solved successively for $b_{1}, \ldots, b_{m-1}$ since $\mu_{m} \neq 0$, while $b_{m}, \ldots, b_{n}$ can be given arbitrary values. 
EXAMPLE 5. Let $G$ be a compact abelian group and let $A=L^{2}(G)$ be the set of functions on $G$ which are square-integrable with respect to Haar measure. Then $A$ is an algebra under convolution. Let $\hat{G}$ denote the dual group and let $\hat{f}$ denote the Fourier transform of a function $f$ on $G$, that is, $\hat{f}(\gamma)=\int f(x) \overline{\gamma(x)} d x$ for all $\gamma \in \hat{G}$.

If $E$ is any subset of $\hat{G}$, then $I_{E}=\{f \in A: \hat{f}(\gamma)=0$ if $\gamma \notin E\}$ is a closed ideal in $A$, and every closed ideal has this form (Hewitt and Ross [5, p. 452, Theorem (38.7)].) The space $L^{2}(G)$ is its own dual, each $\mu \in L^{2}(G)$ defining a linear functional (also denoted $\mu$ ) on $L^{2}(G)$ by $\mu(f)=\int_{G} f(x) \overline{\mu(x)} d x$. We summarize the results on this algebra as follows: Suppose $\mu, \nu \in L^{2}(G)$.

(a) If $E \subset \hat{G}$ and $I=\{f \in A: \hat{f}=0$ off $E\}$ then $\|\mu\|_{I^{*}}=$ $\left(\Sigma_{\gamma \in E}|\hat{\mu}(\gamma)|^{2}\right)^{1 / 2}$.

(b) $\nu \ll \mu$ if and only if $\operatorname{supp}(\hat{\nu}) \subset \operatorname{supp}(\hat{\mu}) .(B y \operatorname{supp}(\hat{\mu})(=$ support of $\mu)$, we mean $\{\gamma \in \hat{G}: \hat{\mu}(\gamma) \neq 0\}$.)

(c) $\nu<<<\mu$ if and only if there exists $K$ such that $|\hat{\nu}(\gamma)| \leqslant K|\hat{\mu}(\gamma)|$ for all $\gamma \in \hat{G}$.

(d) $f \mu=f^{*} * \mu$ for every $f \in A$, where $f^{*}(x)=\overline{f(-x)}$.

(e) The principal band generated by $\mu$ is $\left\{\nu \in L^{2}(G): \hat{\nu}=0\right.$ off $\left.\operatorname{supp}(\hat{\mu})\right\}$. This band is the closure of $\{f \mu: f \in A\}$.

(f) The bands in $L^{2}(G)$ are precisely the closed ideals. Every band is principal if $\hat{G}$ is countable.

To prove (a), note that for every $f \in I$, we have $|\mu(f)|=|S \bar{\mu}|=\left|\Sigma_{\gamma \in E} \hat{f}(\gamma) \overline{\hat{\mu}}(\gamma)\right| \leqslant\left(\sum_{\gamma \in E}|\hat{f}(\gamma)|^{2}\right)^{1 / 2}\left(\Sigma_{\gamma \in E}|\hat{\mu}(\gamma)|^{2}\right)^{1 / 2}$, which implies that $\|\mu\|_{I^{*}}<\left(\sum_{\gamma \in E}|\hat{\mu}(\gamma)|^{2}\right)^{1 / 2}$. To prove the reverse inequality, define $f \in L^{2}(G)$ so that $\hat{f}(\gamma)=\hat{\mu}(\gamma)$ if $\gamma \in E$ and $\hat{f}(\gamma)=0$ if $\gamma \notin E$. Then $\Sigma_{\gamma \in E}|\hat{\mu}(\gamma)|^{2}=\Sigma \hat{f}(\gamma) \hat{\mu}(\gamma)=\mu(f) \leqslant\|f\|\|\mu\|_{I^{*}}=\|\mu\|_{I^{*}} \Sigma_{\gamma \in E}\left(|\hat{\mu}(\gamma)|^{2}\right)^{1 / 2}$, as wanted.

To prove (b), suppose that $\operatorname{supp}(\hat{\nu}) \subset \operatorname{supp}(\hat{\mu})$. Let $\varepsilon>0$. There exists $F=\left\{\gamma_{1}, \ldots, \gamma_{n}\right\}$ such that $\Sigma_{\gamma \notin F}|\hat{\nu}(\gamma)|^{2}<\varepsilon^{2}$ and $\hat{\nu}\left(\gamma_{j}\right) \neq 0$ for each $j$. Let $\delta=\inf \left\{\left|\hat{\mu}\left(\gamma_{j}\right)\right|: j=1, \ldots, n\right\}$. If $I=\{f: \hat{f}=0$ off $E\}$ is an ideal and $\|\mu\|_{I^{*}}<\delta$, then $\Sigma_{\gamma \in E}|\hat{\mu}(\gamma)|^{2}<\delta^{2}$, so that $\gamma_{j} \notin E$ for all $j$ and $\|\nu\|_{\Gamma^{*}}^{2}=$ $\Sigma_{\gamma \in E}|\hat{\nu}(\gamma)|^{2}<\Sigma_{\gamma \notin F}|\hat{\nu}(\gamma)|^{2}<\varepsilon^{2}$, as wanted. The converse of $(\mathrm{b})$ is obvious, as is (c).

To prove (d), we have

$$
\begin{aligned}
(f \mu)(g) & =\mu(f * g)=\int f^{*} g(x) \overline{\mu(x)} d x=\iint f(x-y) g(y) d y \overline{\mu(x)} d x \\
& =\int g(y) \int f(x-y) \overline{\mu(x)} d x d y=\int g(y) \int \overline{f^{*}(y-x) \mu(x)} d x d y \\
& =\int g(y) \overline{\left(f^{*} * \mu\right)(y)} d y .
\end{aligned}
$$

The first statement in (e) is a restatement of (b). To prove the second, note 
that if $\gamma \in \operatorname{supp}(\hat{\mu})$ and $f(x)=\gamma(x)$, then $f \mu$ is the function $f^{*} * \mu(x)=\int \overline{f(y-x)} \mu(y) d y=\int \overline{\gamma(y-x)} \mu(y) d y=\gamma(x) \hat{\mu}(\gamma)$, that is, $\gamma \mu$ $=\hat{\mu}(\gamma) \gamma$. Therefore $\{f \mu: f \in A\}$ includes all trigonometric polynomials which are linear combinations of characters chosen from $\operatorname{supp}(\hat{\mu})$. Now if $\operatorname{supp}(\hat{\nu}) \subset \operatorname{supp}(\hat{\mu})$ then $\nu$ is the $L^{2}$-limit of a sequence of such polynomials (its Fourier series, for example).

To prove (f), recall that every ideal has the form $\left\{\mu \in L^{2}(G): \hat{\mu}=0\right.$ off $E$ \}, for some $E \subset \hat{G}$, and must be a band by (b). Conversely, if $B$ is a band, we let $E=\{\gamma \in \hat{G}: \hat{\mu}(\gamma)=0$ for every $\mu \in B\}$. If $\gamma \notin E$, then $\hat{\mu}(\gamma)=1$ for some $\mu \in B$ and, as before, $\gamma=\hat{\mu}(\gamma) \gamma=\gamma \mu \in B$. Thus $B$ contains all linear combinations of characters chosen from the complement of $E$, so that $B=I_{E}$.

Finally, to prove the last part of (f), suppose that $\hat{G}$ is countable, let $B=I_{E}$ be a band, and let $E=\left\{\gamma_{1}, \gamma_{2}, \gamma_{3}, \ldots\right\}$. Let $\mu(x)=\sum_{n=1}^{\infty} n^{-1} \gamma_{n}(x)$. Then $\operatorname{supp}(\hat{\mu})=E$ and $B$ is the principal band generated by $\mu$.

EXAMPLE 6. Let $G$ be a compact abelian group. Suppose $1<p<\infty$, $p \neq 2$, and consider the algebra $A=L^{p}(G)$ under convolution. The dual of $A$ is $L^{q}(G)$, where $1 / p+1 / q=1$, each $\mu \in A^{*}$ corresponding to a $\mu \in L^{q}$ such that $\mu(f)=\int f \bar{\mu} d x, f \in A$. As in the case $p=2$, the ideals in $A$ have the form $I_{E}=\{f \in A: \hat{f}=0$ off $E\}, E \subset \hat{G}$, but the quantity $\|\mu\|_{I^{*}}$ is not as easily calculated. However, if $E$ has one point $\gamma_{0}$, then $\|\mu\|_{I^{*}}=\left|\hat{\mu}\left(\gamma_{0}\right)\right|$. It follows that if $\nu<<<\mu$ then there is a constant $K$ such that $|\hat{\nu}(\gamma)|<$ $K|\hat{\mu}(\gamma)|$ for all $\gamma \in \hat{G}$, but the converse is not clear. It also follows that if $\nu \ll \mu$ then $\operatorname{supp}(\hat{\nu}) \subset \operatorname{supp}(\hat{\mu})$. The converse of this statement is true. For suppose $\operatorname{supp}(\hat{\nu}) \subset \operatorname{supp}(\hat{\mu})$. As in Example 5, we see that $f \mu=f^{*} * \mu$ for every trigonometric polynomial $f$, so that the band generated by $\mu$ contains all linear combinations of characters in $\operatorname{supp}(\hat{\mu})$. Since $\nu$ is the $L^{q}$-limit of such polynomials, we have $\nu \ll \mu$. Note that this argument also shows that $\{\nu$ : $\nu \ll \mu\}$ is the closure of $\{f \mu: f \in A\}$. As in Example 5, the bands are the closed ideals in $L^{q}(G)$.

EXAMPLE 7. Let $G$ be a compact abelian group and consider the algebra $A=L^{1}(G)$ under convolution. Its dual is $L^{\infty}(G)$. As in previous examples, $\nu<<<\mu$ implies $|\hat{\nu}(\gamma)| \leqslant K|\hat{\mu}(\gamma)|$ for some $K$ and all $\gamma, \mu \ll \nu$ implies $\operatorname{supp}(\hat{\nu}) \subset \operatorname{supp}(\hat{\mu})$, and every band in $L^{\infty}(G)$ is a closed ideal, but the converses of these three statements are not clear. Furthermore, $f \mu=f^{*} * \mu$ if $f \in L^{1}$ and $\mu \in L^{\infty}$, from which it follows that the closure of $\{f \mu: f \in A\}$ can contain only continuous functions, for any $\mu \in L^{\infty}(G)$. In particular, if $\mu$ is not continuous, then the closure of $\{f \mu: f \in A\}$ cannot be the principal band generated by $\mu$.

EXAMPLE 8. Let $A=C^{1}[0,1]$ be the set of functions defined on the interval $[0,1]$ which have one continuous derivative. For any function $f$ on $[0,1]$ we let $\|f\|_{\infty}=\sup \{|f(x)|: 0 \leqslant x \leqslant 1\}$ and if $f \in A$ we let $\|f\|=\|f\|_{\infty}+$ 
$\left\|f^{\prime}\right\|_{\infty}$. With the norm $\|\cdot\|, A$ is a Banach algebra under pointwise multiplication.

From now on, $\int_{a}^{b}$ refers to an integral over the closed interval $[a, b]$. If limits of integration are not explicitly stated, then the interval $[0,1]$ is implied.

If $\mu$ is a finite Borel measure on $[0,1]$ and $a$ is a constant, then $L(f)=$ $a f(0)+\int f^{\prime} d \mu$ clearly defines a bounded linear functional on $A$. Conversely, every bounded linear functional $L$ has this form. For, given $L$, we can find measures $\mu_{1}$ and $\mu_{2}$ so that $L(f)=\int f d \mu_{1}+\int f^{\prime} d \mu_{2}$. But Fubini's theorem gives

$$
\begin{aligned}
\int f d \mu_{1} & =\int_{0}^{1}\left[\int_{0}^{x} f^{\prime}(t) d t+f(0)\right] d \mu_{1}(x) \\
& =f(0) \int_{0}^{1} d \mu_{1}(x)+\int_{0}^{1}\left(\int_{t}^{1} d \mu_{1}(x)\right) f^{\prime}(t) d t
\end{aligned}
$$

so that

$$
L(f)=f(0) \int_{0}^{1} d \mu_{1}+\int_{0}^{1} f^{\prime}(t)\left[\left(\int_{t}^{1} d \mu_{1}(x)\right) d t+d \mu_{2}(t)\right],
$$

as wanted.

Furthermore, the representation $L(f)=a f(0)+\int f^{\prime} d \mu$ is unique. For if $a f(0)+\int f^{\prime} d \mu=0$ for all $f$, then choosing $f(x)=1$ gives $a=0$, and choosing $f(x)=x^{n}, n \geqslant 1$, gives $\int x^{n-1} d \mu(x)=0$ for $n \geqslant 1$, so that $\mu=0$.

We need to know the norm of $L$ on certain ideals. The calculations will include the following notation. If $U=(a, b)$ is an open interval inside $[0,1]$, then the unit triangle function on $U$ is the continuous function $f$ on $[0,1]$ defined as follows: $f(x)=0$ if $x<a$ or $x>b ; f((a+b) / 2)=2 /(b-a)$; and $f$ is linear on the intervals $[a,(a+b) / 2]$ and $[(a+b) / 2, b]$. If $U=$ $\{x: 0<x<b\}$ then the unit triangle function $f$ on $U$ is defined as follows: $f(x)=0$ if $x \geqslant b ; f(0)=2 / b ; f$ is linear on $[0, b]$. The unit triangle function on $U=\{x: a<x<1\}$ is defined as in the previous case. Note that $\int f d x=1$ for any unit triangle function.

Proposition 3. Let a be a constant and $\mu$ a finite measure on $[0,1]$. Define $L$ on $A$ by $L(f)=a f(0)+\int f^{\prime} d \mu, f \in A$.

1. Suppose that $U_{1}, U_{2}, \ldots, U_{n}$ is a family of disjoint open intervals in $[0,1]$ and let $U=\cup_{j=1}^{n} U_{j}$. Let $f_{j}$ be the unit triangle function on $U_{j}$. Let $I=\{f \in$ $A: f(x)=0$ if $x \notin U\}$, which is clearly an ideal in $A$. Then

$$
\|L\|_{I^{*}} \leqslant \sum_{j=1}^{n}\left|d \mu-\left(\int f_{j} d \mu\right) d x\right|\left(U_{j}\right) \leqslant 6\|L\|_{I^{* *}}
$$

Furthermore, for any constants $c_{1}, \ldots, c_{n}$ 


$$
\|L\|_{I^{*}}<\sum_{j=1}^{n}\left|d \mu-c_{j} d x\right|\left(U_{j}\right) .
$$

2. Suppose that $U=[0, t), I=\{f \in A: f(x)=0$ if $x>t\}$. Then

$$
\|L\|_{I^{*}}<|d \mu-a d x|([0, t))<2\|L\|_{I^{*}}
$$

3. Suppose that $U=(t, 1], I=\{f \in A: f(x)=0$ if $x<t\}$. Then

$$
\|L\|_{I^{*}}<|d \mu|((t, 1])<2\|L\|_{I^{*}}
$$

Proof. First we prove (1). Let $f$ be any continuous function on $[0,1]$ such that $f(x)=0$ if $x \notin U$. Define $g$ on $[0,1]$ by

$$
g(x)=\int_{0}^{x}\left[f(t)-\sum_{j=1}^{n}\left(\int_{U_{j}} f(x) d s\right) f_{j}(t)\right] d t .
$$

Then $g \in I$ and $g^{\prime}(x)=f(x)-\sum_{j=1}^{n}\left(\int_{U_{j}} f(s) d s\right) f_{j}(x)$. Also, if $x \in U_{j}$, then (letting $\left|U_{j}\right|$ denote the length of $U_{j}$ ) we have

$$
\begin{aligned}
\left|g^{\prime}(x)\right| & =\left|f(x)-f_{j}(x) \int_{U_{j}} f(s) d s\right| \leqslant\|f\|_{\infty}+\left\|f_{j}\right\|_{\infty}\left|U_{j}\right|\|f\|_{\infty} \\
& =\|f\|_{\infty}+2\|f\|_{\infty}=3\|f\|_{\infty},
\end{aligned}
$$

so that $\left\|g^{\prime}\right\|_{\infty}<3\|f\|_{\infty}$. Since $g$ is 0 at some point, we can write $g$ as the integral of $g^{\prime}$ and get $\|g\|_{\infty}<\left\|g^{\prime}\right\|_{\infty}<3\|f\|_{\infty}$, so that $\|g\|<6\|f\|_{\infty}$. Now $|L(g)|<\|g\|\|L\|_{I^{*}}<6\|f\|_{\infty}\|L\|_{I^{*}}$, and

$$
\begin{aligned}
L(g) & =\int_{0}^{1}\left[f(x)-\sum_{j=1}^{n}\left(\int_{U_{j}} f(s) d s\right) f_{j}(x)\right] d \mu(x) \\
& =\sum_{j=1}^{n} \int_{U_{j}} f(x) d \mu(x)-\sum_{j=1}^{n} \int_{U_{j}} f(s) d s \int f_{j}(x) d \mu(x) \\
& =\sum_{j=1}^{n} \int_{U_{j}} f(s)\left[d \mu(s)-\left(\int f_{j}(x) d \mu(x)\right) d s\right] .
\end{aligned}
$$

Therefore we have shown

$$
\left|\sum_{j=1}^{n} \int_{U_{j}} f(s)\left[d \mu(s)-\left(\int f_{j}(x) d \mu(x)\right) d s\right]\right|<6\|f\|_{\infty}\|L\|_{I^{*}}
$$

for any continuous function $f$ on $[0,1]$ which is 0 off $U$. This implies the second inequality in (1).

The inequality in (2) is easier. Suppose that $g \in I$. Then $\int_{v,} g^{\prime}(x) d x=0$ because $g$ is 0 at the endpoints of $U_{j}$. Therefore for any constants $c_{j}$, we have 


$$
\begin{aligned}
|L(g)| & =\left|\int g^{\prime} d \mu\right|=\left|\sum_{j=1}^{n} \int_{U_{j}} g^{\prime}(x) d \mu(x)\right| \\
& =\left|\sum_{j=1}^{n} \int_{U_{j}} g^{\prime}(x)\left[d \mu(x)-c_{j} d x\right]\right|<\sum_{j=1}^{n}\|g\|\left|d \mu-c_{j} d x\right|\left(U_{j}\right),
\end{aligned}
$$

so that $\|L\|_{I^{*}} \leqslant \sum_{j=1}^{n}\left|d \mu-c_{j} d x\right|\left(U_{j}\right)$ for any $c_{j}$. Choosing $c_{j}=\int f_{j} d \mu$, we get (1).

The proofs of (3) and (4) are similar.

Suppose that $\mu$ is a measure on $[0,1]$. We say that $\mu$ is constant on a set $E$ if there is a constant $c$ such that $d \mu=c d x$ on $E$. We can write $\mu$ in the form $\mu=\mu_{a}+\mu_{s}$ where $\mu_{a}$ is absolutely continuous (in the usual measure theoretic sense) with respect to Lebesgue measure and $\mu_{s}$ is singular with respect to Lebesgue measure. We shall refer to $\mu_{a}$ and $\mu_{s}$ as the absolutely continuous and singular parts of $\mu$, respectively.

Proposition 4. Suppose that $L, G \in A^{*}=C^{1^{*}}$ are given by $L(f)=a f(0)$ $+\int f^{\prime} d \mu$ and $G(f)=b f(0)+\int f^{\prime} d \nu$, for constants $a$ and $b$ and measures $\mu$ and v. Then $G \ll L$ if and only if each of the following conditions is satisfied:

1. If $0<\alpha<\beta<1$ and $\mu$ is constant on $(\alpha, \beta)$, then $\nu$ is constant on $(\alpha, \beta)$.

2. If $d \mu=a d x$ on $[0, \beta)$ then $d \nu=b d x$ on $[0, \beta)$. If $d \mu=0$ on $(\alpha, 1]$ then $d \nu=0$ on $(\alpha, 1]$.

3. The singular part of $\nu$ is absolutely continuous (in the measure theoretic sense) with respect to the singular part of $\mu$.

Furthermore, if $G \ll L$, then $G$ is the (norm) limit of linear functionals of the form $g L$, where $g \in A$.

Before proving this, we would like to note that it contains the classical Riemann-Lebesgue Lemma.

COROLLARY. Suppose that $h$ is Lebesgue integrable on $[0,1]$. Then

$$
\lim _{n \rightarrow \infty} \int_{0}^{1} h(x) \sin 2 \pi n x d x=\lim _{n \rightarrow \infty} \int_{0}^{1} h(x) \cos 2 \pi n x d x=0 .
$$

Proof. Define $G$ and $L$ on $A$ as follows: $G(f)=\int h(x) f^{\prime}(x) d x$ and $L(f)=\int_{0}^{1} f(x) d x$. Then

$$
\begin{aligned}
L(f) & =\int_{0}^{1}\left[\int_{0}^{x} f^{\prime}(t) d t+f(0)\right] d x \\
& =\int_{0}^{1}\left(\int_{t}^{1} d x\right) f^{\prime}(t) d t+f(0)=\int_{0}^{1}(1-t) f^{\prime}(t) d t+f(0) .
\end{aligned}
$$

By the previous proposition, $G \ll L$.

Now let $I_{n}=\{f \in A: f(j / n)=0$ for $j=0,1,2, \ldots, n\}$. If $f \in I_{n}$ and 
$\|f\|<1$ then clearly $\|f\|_{\infty} \leqslant 1 / n$ and $|L(f)| \leqslant 1 / n$. That is, $\|L\|_{I_{n}^{*}}<1 / n$. Therefore $\|G\|_{I_{n}^{*}} \rightarrow 0$ as $n \rightarrow \infty$. But if $f_{n}(x)=(\sin 2 \pi n x) / 2 \pi n$, then $f_{n} \in I_{n}$ and $\left\|f_{n}\right\|<2$, so $\left|G\left(f_{n}\right)\right|=\left|\int h(x) \cos 2 \pi n x d x\right| \rightarrow 0$ as $n \rightarrow \infty$. The proof for sin is similar.

Proof of Proposition 4. First we will assume $G \ll L$ and prove that the three conditions are satisfied. Suppose that $d \mu=c d x$ on $(\alpha, \beta)$. If $I=\{f \in$ $A: f(x)=0$ if $x \notin(\alpha, \beta)\}$ and $f$ denotes the unit triangle function on $(\alpha, \beta)$, then $\int f d \mu=c \int f d x=c$ and by Proposition $3\|L\|_{I^{*}}<\left|d \mu-\left(\int f d \mu\right) d x\right|(U)$ $=0$. Therefore $\|G\|_{I^{*}}=0$, so that $\left|d \nu-\left(\int f d \nu\right) d x\right|(U)=0$, that is $d \nu=$ $\left(\int f d v\right) d x$ on $U$. Thus the first condition is satisfied, and in the same way we can prove the second.

To prove the third, let $\sigma$ and $\tau$ denote the singular parts of $\mu$ and $\nu$. Note that it suffices to prove that $\tau$ is absolutely continuous with respect to $\mu$. For, we know there exists a set $T$ of Lebesgue measure 0 such that $|\tau|([0,1]-T)$ $=0$. Now suppose $S$ is any set such that $|\sigma|(S)=0$. Then $T \cap S$ has Lebesgue measure 0 and $\sigma$-measure 0 , hence $\mu$-measure 0 , hence $\tau$ measure 0 , so $|\tau|(S)=|\tau|(S \cap T)+|\tau|(S-T)=0+0$.

So we have to show that if $|\mu|(E)=0$ then $|\tau|(E)=0$. Let us also assume $0 \notin E$. Let $\varepsilon>0$. Choose $\delta>0$ so that $\|G\|_{I^{*}}<\varepsilon$ whenever $\|L\|_{I^{*}}<\delta$. Choose a sequence $U_{1}, U_{2}, \ldots$ of disjoint open intervals such that $E \subset$ $\cup_{j=1}^{\infty} U_{j}, \sum_{j=1}^{\infty}|\mu|\left(U_{j}\right)<\delta$, and $0 \notin \cup_{j=1}^{\infty} U_{j}$. If $I_{n}=\{f \in A: f(x)=0$ if $\left.x \notin \cup{ }_{j=1}^{n} U_{j}\right\}$, then clearly $\|L\|_{I_{n}^{*}}<\delta$, so that $\|G\|_{I_{n}^{*}}<\varepsilon$. By Proposition 3 we have

$$
\sum_{j=1}^{n}\left|d \nu-\left(\int f_{j} d \nu\right) d x\right|\left(U_{j}\right)<6\|G\|_{I^{*}}<6 \varepsilon,
$$

where $f_{j}$ denotes the unit triangle function on $U_{j}$. Considering singular parts, we get $|\tau|\left(\cup_{j=1}^{n} U_{j}\right)<6 \varepsilon$. Letting $n \rightarrow \infty$ implies $|\tau|(E)<6 \varepsilon$, and since $\varepsilon>0$ is arbitrary we have $|\tau|(E)=0$, as wanted.

To remove the assumption $0 \notin E$ we must show that if $\mu$ has no point mass at 0 , then $\nu$ has none either. If $I_{\varepsilon}=\{f \in A: f(x)=0$ if $x>\varepsilon\}$, then $\|L\|_{I_{i}^{*}}<|A| \varepsilon+|\mu|([0, \varepsilon])$, which goes to 0 as $\varepsilon>0$ if $\mu$ has no mass at 0 . Suppose now that $\nu$ has mass $c$ at 0 , that is $\nu(\{0\})=c$. Let $\delta_{0}$ denote the unit mass at 0 , and let $\nu_{0}=\nu-c \delta_{0}$, so that $\nu_{0}$ has no mass at 0 . Choose a function $f_{e} \in I_{\varepsilon}$ so that $f_{e}^{\prime}(0)=1$ and $\left\|f_{e}^{\prime}\right\|_{\infty}=1$. Then $\left|f_{\varepsilon}(0)\right|<\varepsilon$ and

$$
\begin{aligned}
2\|G\|_{I_{\varepsilon}} \geqslant\left|G\left(f_{\varepsilon}\right)\right| & =\left|b f_{\varepsilon}(0)+\int f_{\varepsilon}^{\prime} d \nu\right| \\
& =\left|b f_{\varepsilon}(0)+c f_{\varepsilon}^{\prime}(0)+\int f_{\varepsilon}^{\prime} d \nu_{0}\right| \\
& \geqslant|c|-b \varepsilon-\left|\nu_{0}\right|([0, \varepsilon]) .
\end{aligned}
$$


The last term converges to $|c|$ as $\varepsilon \rightarrow 0$, so if $c \neq 0$ then $\|G\|_{r_{\varepsilon}^{*}}$ does not go to 0 as $\varepsilon \rightarrow 0$, contradicting $G \ll L$. So the third condition is proven.

Conversely, suppose that the three conditions hold.

First, we must find an expression for $g L$ when $g \in A$. We have $(g L)(f)=$ $L(f g)=a f(0) g(0)+\int f^{\prime} g d \mu+\int f g^{\prime} d \mu$. Writing $f$ as the integral of $f^{\prime}$ gives

$$
\int f g^{\prime} d \mu=f(0) \int g^{\prime} d \mu+\int f^{\prime}(x)\left[\int_{x}^{1} g^{\prime}(t) d \mu(t)\right] d x
$$

so that

$$
\begin{aligned}
(g L)(f)= & f(0)\left[a g(0)+\int g^{\prime} d \mu\right] \\
& +\int f^{\prime}(x)\left[\left(\int_{x}^{1} g^{\prime}(t) d \mu(t)\right) d x+g(x) d \mu(x)\right] .
\end{aligned}
$$

Suppose that $d \mu=u(x) d x+d \sigma$, where $\sigma$ is the singular part of $\mu$ and $u$ is Lebesgue integrable. Then

$$
\begin{aligned}
(g L)(f)= & f(0)\left[a g(0)+\int_{0}^{1} g^{\prime} d \mu\right] \\
& +\int_{0}^{1} f^{\prime}(x)\left[\left(\int_{x}^{1} g^{\prime}(t) d \mu(t)+g(x) u(x)\right) d x+g(x) d \sigma(x)\right] .
\end{aligned}
$$

Consider the Banach space direct sum $S=\mathbf{C} \oplus L^{1}(d x) \oplus L^{1}(d \sigma)$. Let $B$ be the subspace of all triples $\left\langle d, l_{1}, l_{2}\right\rangle$ in $S$ such that

(i) $l_{1}$ is constant on every subinterval $(\alpha, \beta)$ of $[0,1]$ on which $d \mu$ is a constant,

(ii) $l_{1}=d$ on $[0, \beta)$ if $d \mu=a d x$ on $[0, \beta)$, and $l_{1}=0$ on $(\alpha, 1]$ if $d \mu=0$ on $(\alpha, 1]$.

Let $C$ be the subspace of all triples in $S$ of the form

$$
\left\langle a g(0)+\int_{0}^{1} g^{\prime} d \mu, \int_{x}^{1} g^{\prime} d \mu+g(x) u(x), g(x)\right\rangle, \quad g \in A .
$$

It is easy to see that $C \subset B$.

We will show that $C$ is dense in $B$. Once we have shown this, we complete the proof as follows. Suppose that the three conditions of the proposition are satisfied and $d \nu=l_{1} d x+d \tau$ and $\tau$ is the singular part of $\nu$ and $l_{1}$ is Lebesgue integrable. By the third condition, we have $d \tau=l_{2} d \sigma$ for some $l_{2} \in L^{1}(d \sigma)$, so that $G(f)=a f(0)+\int f^{\prime}(x)\left[l_{1}(x) d x+l_{2}(x) d \sigma(x)\right]$ and the first two conditions imply that $\left\langle b, l_{1}, l_{2}\right\rangle \in B$. Hence there exists a sequence $g_{n} \in C^{1}$ such that

$$
\left\{a g_{n}(0)+\int_{0}^{1} g_{n}^{\prime} d \mu, \int_{x}^{1} g_{n}^{\prime} d \mu+g_{n}(x) u(x), g_{n}(x)\right\rangle \rightarrow\left\langle b, l_{1}, l_{2}\right\rangle .
$$


Therefore $g_{n} L \rightarrow G$. Since $g_{n} L \ll L$, this proves $G \ll L$. It also proves the last statement of the proposition.

To show that $C$ is dense in $B$ we will show that every element of $S^{*}$ which annihilates $C$ also annihilates $B$. An element of $S^{*}$ can be assumed to be a triple $\left\langle c, k_{1}, k_{2}\right\rangle$ in $\mathbf{C} \oplus L^{\infty}(d x) \oplus L^{\infty}(d \sigma)$. If this element annihilates $C$, we have

$$
\begin{aligned}
{\left[a g(0)+\int g^{\prime} d \mu\right] c+\int\left[\int_{x}^{1} g^{\prime} d \mu+g(x) u(x)\right] k_{1}(x) d x } & \\
& +\int g(x) k_{2}(x) d \sigma(x)=0
\end{aligned}
$$

or

$$
\begin{aligned}
\operatorname{cag}(0)+\int g^{\prime}(t)[c+ & \left.\int_{0}^{t} k_{1}(x) d x\right] d \mu(t) \\
& +\int g(x)\left[u(x) k_{1}(x) d x+k_{2}(x) d \sigma(x)\right]=0
\end{aligned}
$$

for all $g \in A$. Letting $d \lambda=u(x) k_{1}(x) d x+k_{2}(x) d \sigma(x)$, the last integral becomes $\int g(x) d \lambda(x)=g(0) \int_{0}^{1} d \lambda+\int g^{\prime}(t)\left[\int_{t}^{1} d \lambda(x)\right] d t$ and substituting in (5) gives

$$
\left[c a+\int_{0}^{1} d \lambda\right] g(0)+\int g^{\prime}(t)\left[\left(c+\int_{0}^{t} k_{1}(x) d x\right) d \mu(t)+\left(\int_{t}^{1} d \lambda(x)\right) d t\right]=0
$$

for all $g \in A$. It follows that the coefficient of $g(0)$ as well as the measure in the integral in (6) must be 0 . Breaking this measure into absolutely continuous and singular parts, we get three equations from (6):

$$
\begin{gathered}
c a+\int_{0}^{1}\left[u(x) k_{1}(x) d x+k_{2}(x) d \sigma(x)\right]=0, \\
\left(c+\int_{0}^{t} k_{1}(x) d x\right) u(t)+\int_{t}^{1}\left[u(x) k_{1}(x) d x+k_{2}(x) d \sigma(x)\right]=0 \\
\left(c+\int_{0}^{t} k_{1}(x) d x\right) d \sigma=0 .
\end{gathered}
$$

Consider the continuous function $c+\int_{0}^{t} k_{1}(x) d x$ and let $K=\{t \in[0,1]$ : $\left.c+\int_{0}^{t} k_{1}(x) d x=0\right\}$. We claim that $k_{1}=0$ a.e. $(d x)$ on $K$. For, almost every $(d x)$ point $t \in K$ is a point of density of $K$ such that

$$
k_{1}(t)=\lim \frac{1}{\varepsilon} \int_{t}^{t+e} k_{1}(x) d x .
$$


But if $\varepsilon$ is chosen so that $t+\varepsilon \in K$ then

$$
\int_{t}^{t+\varepsilon} k_{1}(x) d x=c+\int_{0}^{t+\varepsilon} k_{1}(x) d x-\left[c+\int_{0}^{t} k_{1}(x) d x\right]=0,
$$

so $k_{1}(t)=0$.

Now suppose that $(\gamma, \delta)$ is disjoint from $K$. We claim that $d \mu$ is a constant on $(\gamma, \delta)$. By (9), $\sigma=0$ on $(\gamma, \delta)$. Hence the integral $\int_{t}^{1} k_{2}(x) d \sigma(x)$ in (8) is constant for $t \in(\gamma, \delta)$. The other integrals in (8) are (on compact subsets) absolutely continuous functions of $t$, and since its coefficient is not zero $u$ is also absolutely continuous on $(\gamma, \delta)$. So $(8)$ can be differentiated to get

$$
k_{1}(t) u(t)+\left(c+\int_{0}^{t} k_{1}(x) d x\right) u^{\prime}(t)-u(t) k_{1}(t)=0 \text { for a.e. } t \in(\gamma, \delta) .
$$

Hence $u^{\prime}(t)=0$ for a.e. $t \in(\gamma, \delta)$, so that $u$ is constant on $(\gamma, \delta)$. Since $\sigma=0$ on $(\gamma, \delta)$, we have $d \mu$ is constant on $(\gamma, \delta)$.

Recall that we are trying to show that $\left\langle c, k_{1}, k_{2}\right\rangle$ annihilates $B$. Let us assume for the moment that condition (ii) in the definition of $B$ does not apply. We can find a sequence of disjoint intervals $\left(\alpha_{n}, \beta_{n}\right)$ such that $d \mu$ is constant on $\left(\alpha_{n}, \beta_{n}\right), d \mu$ is not constant on any open interval containing $\left(\alpha_{n}, \beta_{n}\right)$, and $d \mu$ is not constant on any open interval except those contained in some $\left(\alpha_{n}, \beta_{n}\right)$. Let $N=\left\{x \in[0,1]: x \notin\left(\alpha_{n}, \beta_{n}\right)\right.$ for all $\left.n\right\}$. Then $B$ consists of triples $\left\langle d, l_{1}, l_{2}\right\rangle$ such that $l_{1}$ is constant on each $\left(\alpha_{n}, \beta_{n}\right)$, with no restriction on $d$ and $l_{2}$. To show that $\left\langle c, k_{1}, k_{2}\right\rangle$ annihilates $B$ we must show that $c=0$, that $k_{2}=0$, that $k_{1}(x)=0$ for $x \in N$, and that $\int_{\alpha_{n}}^{\beta_{n}} k_{1}(x) d x=0$ for all $n$.

We claim $\alpha_{n}, \beta_{n} \in K$ for all $n$. First suppose $\alpha_{n} \neq 0$. Now if $\alpha_{n} \notin K$, then there exist $\gamma$ and $\delta$ such that $\alpha_{n} \in(\gamma, \delta) \subset[0,1]$ and $(\gamma, \delta)$ is disjoint from $K$. But we have shown that $\mu$ is a constant on $(\gamma, \delta)$, hence on $\left(\gamma, \beta_{n}\right)$, contradicting the maximality of $\left(\alpha_{n}, \beta_{n}\right)$. Hence $\alpha_{n} \in K$, as wanted. Next suppose $\alpha_{n}=0$. We are assuming that $u$ is a constant (say $\left.u_{0}\right)$ on $\left(0, \beta_{n}\right)$ and that $\sigma=0$ on $\left(0, \beta_{n}\right)$. Since we are also assuming that (ii) does not apply, there are two possibilities: either $\sigma$ as a nonzero point mass at 0 , or else $\sigma=0$ on $\left[0, \beta_{n}\right)$ but $u_{0} \neq a$. In the first case, (9) implies $c=0$, so that clearly $\alpha_{n}=0 \in K$. In the second case, if we choose $t$ so that $0<t<\beta_{n}$ we have $\int_{0}^{t} k_{1}(x) d x u(t)=$ $\int_{0}^{t} u(x) k_{1}(x) d x$ and $\int_{t}^{1} k_{2}(x) d \sigma(x)=\int_{0}^{1} k_{2}(x) d \sigma(x)$, so that (8) becomes

$$
c u_{0}+\int_{0}^{1} u(x) k_{1}(x) d x+\int_{0}^{1} k_{2}(x) d \sigma(x)=0 .
$$

Comparing this to (7), we find that $c a=c u_{0}$, and since $a \neq u_{0}$, we have $c=0$. Hence $\alpha_{n}=0 \in K$.

The proof that $\beta_{n} \in K$ is analogous. If $\beta_{n} \neq 1$, it follows as before. If $\beta_{n}=1$, the possibilities are: either $\sigma$ has a nonzero point mass at 1 , or else 
$\sigma=0$ on $\left(\alpha_{n}, 1\right]$ but $u=u_{0} \neq 0$ on $\left(\alpha_{n}, 1\right]$. In the first case, (9) implies $c+\int_{0}^{1} k_{1}(x) d x=0$, or $\beta_{n}=1 \in K$, and in the second case choosing $t \in$ $\left(\alpha_{n}, 1\right)$ in (8) gives $\left(c+\int_{0}^{1} k_{1}(x) d x\right) u_{0}=0$, so again $\beta_{n}=1 \in K$.

Now since $\alpha_{n}, \beta_{n} \in K$, we have $c+\int_{0}^{\alpha_{0}} k_{1}(x) d x=c+\int_{0}^{\beta_{n}} k_{1}(x) d x=0$, so

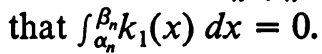

If $x \in N$, then $x \in K$. For if $x \notin K$, then $\mu$ is a constant on an interval around $x$, contradicting the definition of $N$. Hence $N \subset K$, and we already know that $k_{1}$ is 0 a.e. on $K$, hence on $N$.

Furthermore, $c$ must be 0 . If not, then $c+\int_{0}^{t} k_{1}(x) d x \neq 0$ for $t$ near 0 , which we have seen implies that $d \mu$ is a constant on $(0, \delta)$ for some $\delta$. That is, $\alpha_{n}=0$ for some $n$, and we showed that $c=0$ under these circumstances.

Finally, we must show $k_{2} d \sigma=0$. We claim that $\int_{t}^{1} k_{1}(x) u(x) d x=$ $u(t) \int_{t}^{1} k_{1}(x) d x$ for a.e. $t$. Note that $\int_{\alpha_{n}}^{\beta_{n}} k_{1}(x) u(x) d x=\int_{\alpha_{n}}^{\beta_{n}} k_{1}(x) d x=0$ for every $n$ since $u$ is constant on each $\left(\alpha_{n}, \beta_{n}\right)$. Now if $t \in N$, then $[t, 1]$ is the union of a subset of $N$ plus some of the intervals $\left(\alpha_{n}, \beta_{n}\right)$, and $k_{1}=0$ a.e. on $N$, so that $\int_{t}^{1} k_{1}(x) u(x) d x=\int_{t}^{1} k_{1}(x) d x u(t)=0$. On the other hand if $t \notin N$, then $t \in\left(\alpha_{j}, \beta_{j}\right)$ for some $j$, and $\int_{\beta_{j}}^{1} k_{1}(x) u(x) d x=\int_{\beta_{j}}^{1} k_{1}(x) d x=0$ for the reasons just mentioned. Therefore

$$
\int_{t}^{1} k_{1}(x) u(x) d x=\int_{t}^{\beta_{j}} k_{1}(x) u(x) d x=u(t) \int_{t}^{\beta_{j}} k_{1}(x) d x=u(t) \int_{t}^{1} k_{1}(x) d x,
$$

which proves the claim. Hence (8) may be rewritten as

$$
\left(c+\int_{0}^{1} k_{1}(x) d x\right) u(t)+\int_{t}^{1} k_{2}(x) d \sigma(x)=0 \text { for a.e. } t .
$$

Since $c=\int_{0}^{1} k_{1}(x) d x=0$, we have $\int_{t}^{1} k_{2}(x) d \sigma(x)=0$ for a.e. $t$. But $\int_{t}^{1} k_{2}(x) d \sigma(x)$ is continuous in $t$ except perhaps for jumps at the point masses of $\sigma$, so that $\int_{t}^{1} k_{2}(x) d \sigma(x)=0$ for all $t$, which implies $k_{2} d \sigma=0$, as wanted. This completes the proof under the assumption that (ii) does not apply.

To complete the proof of the proposition, suppose that (ii) applies. Let the $\left(\alpha_{n}, \beta_{n}\right)$ and $N$ be defined as before, except that the special intervals $[0, \beta)$ and $(\alpha, 1]$ will not be included among the $\left(\alpha_{n}, \beta_{n}\right)$. Then $B$ consists of triples $\left\langle d, l_{1}, l_{2}\right\rangle$ such that $l_{1}$ is constant on each $\left(\alpha_{n}, \beta_{n}\right), l_{1}=d$ on $(0, \beta)$, and $l_{1}=0$ on $(\alpha, 1]$. Suppose that $\left\langle c, k_{1}, k_{2}\right\rangle$ annihilates $C$. To show that $\left\langle c, k_{1}, k_{2}\right\rangle$ annihilates $B$ it suffices to show that $k_{1}=0$ on $N$, that $\int_{\alpha_{n}}^{\beta_{n}} k_{1}(x) d x$ $=0$ for all $n$, that $c+\int_{0}^{\beta} k_{1}(x) d x=0$, and that $k_{2} d \sigma=0$.

The first two of these statements are proven as before. To prove the third, note that $\beta \in K$ because $d \mu$ is not a constant in a neighborhood of $\beta$. To prove the fourth, note that (8) can be rewritten as

$$
\left(c+\int_{0}^{i} k_{1}(x) d x\right) u(t)+\int_{t}^{\alpha}\left[u(x) k_{1}(x) d x+k_{2}(x) d \sigma(x)\right]=0
$$

for a.e. $t \in[0, \alpha]$. 
As before, we can show

$$
\left(c+\int_{0}^{\alpha} k_{1}(x) d x\right) u(t)+\int_{t}^{\alpha} k_{2}(x) d \sigma(x)=0 \quad \text { for a.e. } t \in[0, \alpha] \text {. }
$$

Since $c+\int_{0}^{\alpha} k_{1}(x) d x=c+\int_{0}^{\beta} k_{1}(x) d x+\int_{\beta}^{\alpha} k_{1}(x) d x=0$, we have $\int_{t}^{\alpha} k_{2}(x) d \sigma(x)=0$ for a.e. $t \in[0, \alpha]$, so that $k_{2} d \sigma=0$ on $[0, \alpha]$. Since $d \sigma=0$ on $[\alpha, 1]$, we are done.

The relations $G<<<L$ appears to be more difficult to characterize. The next few theorms give some results in this direction.

Proposition 5. Suppose that $L(f)=\int f d \mu$ for all $f \in A$, where $\mu$ is a finite Borel measure on $[0,1]$, and that $G<<<L$. Then there exists a finite measure $\nu$ on [0,] such that $G(f)=\int f d \nu$ and $\nu$ is boundedly absolutely continuous (in the usual measure theoretic sense) with respect to $\mu$.

Proof. We can put $L$ in the form

$$
\begin{aligned}
L(f) & =\int_{0}^{1}\left[f(0)+\int_{0}^{x} f^{\prime}(t) d t\right] d \mu(x) \\
& =f(0) \int d \mu+\int_{0}^{1} f^{\prime}(t)\left(\int_{t}^{1} d \mu(x)\right) d t .
\end{aligned}
$$

It follows from Proposition 4 that $G$ has the form $G(f)=a f(0)+\int f^{\prime} g d x$, where $g \in L^{1}(d x)$.

Now suppose that $0<\alpha<\beta<1$ and let $\varepsilon=(\beta-\alpha) / 2$. Choose a sequence $f_{n} \in A$ approximating a right triangle with $(\alpha, \beta)$ as hypotenuse, that is, choose $f_{n} \in A$ so that $\left\|f_{n}^{\prime}\right\|_{\infty}<1, f_{n}(x)=0$ if $x \in[0, \alpha] \cup[\beta, 1]$, and

$$
f_{n}^{\prime}(x) \rightarrow \begin{cases}1 & \text { if } \alpha<x<\alpha+\varepsilon \\ -1 & \text { if } \alpha+\varepsilon<x<\beta\end{cases}
$$

Note that $f_{n} \in I=\{f \in A: f(x)=0$ if $x \notin(\alpha, \beta)\}$ and that $\left\|f_{n}\right\|<2$, so that $\left|G\left(f_{n}\right)\right|<2\|G\|_{I^{*}}$. Now if $f \in I$ and $\|f\|<1$, then $\left\|f^{\prime}\right\|_{\infty}<1$ and $\|f\|_{\infty}<\varepsilon$, so that $|L(f)|=\left|\int f d \mu\right|<\varepsilon|\mu|(\alpha, \beta)$ and $\|L\|_{I^{*}}<\varepsilon|\mu|(\alpha, \beta)$. If now $K$ is a constant such that $\|G\|_{I^{*}}<K\|L\|_{I^{*}}$ for all ideals $I$ in $A$, we have

$$
\left|G\left(f_{n}\right)\right|<2 K \varepsilon|\mu|(\alpha, \beta)
$$

Since $G\left(f_{n}\right)=\int f_{n}^{\prime} g d x$, we may let $n \rightarrow \infty$ in (10) to obtain

$$
\left|\int_{\alpha}^{\alpha+\varepsilon} g d x-\int_{\alpha+\varepsilon}^{\beta} g d x\right|<2 K \varepsilon|\mu|(\alpha, \beta) .
$$

Next suppose that $0<s<t<1$. Let $n$ be a large integer and let $\varepsilon=(t-$ $s) / n$. Then applying (11) repeatedly gives 


$$
\begin{aligned}
\left|\int_{s}^{s+\varepsilon} g d x-\int_{t}^{t+\varepsilon} g d x\right| & =\left|\sum_{j=1}^{n}\left(\int_{s+(j-1) \varepsilon}^{s+j \varepsilon} g d x-\int_{s+j \varepsilon}^{s+(j+1) \varepsilon} g d x\right)\right| \\
& \leqslant \sum_{j=1}^{n} 2 K \varepsilon|\mu|(s+(j-1) \varepsilon, s+(j+1) \varepsilon) \\
& \leqslant 4 K \varepsilon|\mu|(s, t+\varepsilon) .
\end{aligned}
$$

Hence

$$
\left|\frac{1}{\varepsilon} \int_{s}^{s+\varepsilon} g d x-\frac{1}{\varepsilon} \int_{t}^{t+\varepsilon} g d x\right| \leqslant 4 K|\mu|(s, t+\varepsilon) .
$$

Letting $n \rightarrow \infty$, we find that for a.e. $s$ and a.e. $t$ such that $0 \leqslant s<t<1$

$$
|g(s)-g(t)| \leqslant 4 K|\mu|(s, t] \text {. }
$$

We wish to show that $g$ is of bounded variation and determines a measure boundedly absolute continuous with respect to $\mu$. We may assume without loss of generality that $g$ is real-valued. Define

$$
g_{0}(s)=\lim _{t \rightarrow s+} \text { ess } \sup \{g(\alpha): s<\alpha<t\}
$$

for $s \in[0,1)$. From (12) it follows that $g=g_{0}$ a.e. so that (12) holds with $g_{0}$ in place of $g$ and a.e. $s$ and $t$. Furthermore, $g_{0}$ is continuous from the right. Therefore given any $s, t$ such that $0 \leqslant s<t<1$, we can find sequences $s_{n}>s$ and $t_{n}>t$ such that $s_{n} \rightarrow s, t_{n} \rightarrow t$, and (12) holds with $g, s, t$ replaced by $g_{0}, s_{n}, t_{n}$. Letting $n \rightarrow \infty$ then gives

$$
\left|g_{0}(s)-g_{0}(t)\right|<4 K|\mu|(s, t], \quad 0 \leqslant s<t<1 .
$$

It follows that $g_{0}(t)$ has a limit as $t \rightarrow 1$, which we take as the definition of $g_{0}(1)$. From (13) we see that $g_{0}$ is of bounded variation, and there exists a measure $\nu$ on $(0,1]$ such that $\nu(s, t]=g_{0}(t)-g_{0}(s)$ if $0 \leqslant s<t<1$. Note that $\nu$ has no mass at 1 and define $\nu(\{0\})=0$. By (13) $\nu$ is boundedly absolutely continuous with respect to $\mu$ on $[0,1]$. If $f \in A$, then

$$
\begin{aligned}
G(f) & =a f(0)+\int f^{\prime} g_{0} d x=a f(0)+\int g_{0} d f \\
& =a f(0)-\int f d g_{0}+f(1) g_{0}(1)-f(0) g_{0}(0) \\
& =\left(a-g_{0}(0)\right) f(0)+g_{0}(1) f(1)-\int f d \nu .
\end{aligned}
$$

To complete the proof, we only have to show that if $\mu$ has no mass at 0 then $a-g_{0}(0)=0$ and if $\mu$ has no mass at 1 then $g_{0}(1)=0$.

Suppose $\mu$ has no mass at 0 . If $\varepsilon>0$ and $I=\{f \in A: f(x)=0$ if $x \notin[0, \varepsilon)\}$ then $\|f\|_{\infty}<\varepsilon$ whenever $f \in I$ and $\|f\|<1$, so that $\|L\|_{I^{*}}<$ $\varepsilon|\mu|[0, \varepsilon)$. Hence $\|G\|_{I^{*}}<K \varepsilon|\mu|[0, \varepsilon)$. Choose a sequence $f_{n} \in I$ so that 
$\left\|f_{n}^{\prime}\right\|_{\infty}<1, f_{n}(0) \rightarrow \varepsilon$, and $f_{n}^{\prime}(x) \rightarrow-1$ for $x \in[0, \varepsilon)$ as $n \rightarrow \infty$. Then $\left|G\left(f_{n}\right)\right|$ $<\left\|f_{n}\right\|\|G\|_{I^{*}}<2 K \varepsilon|\mu|[0, \varepsilon)$, and letting $n \rightarrow \infty$ gives $\left|a \varepsilon-\int_{0}^{e} g_{0} d x\right|<$ $2 K \varepsilon|\mu|[0, \varepsilon)$. Divide by $\varepsilon$ to get

$$
\left|a-\frac{1}{\varepsilon} \int_{0}^{e} g_{0} d x\right|<2 K|\mu|[0, \varepsilon) .
$$

Since $g_{0}$ is continuous from the right, the integral in (14) goes to $g_{0}(0)$ as $\varepsilon \rightarrow 0$, and since $|\mu|(\{0\})=0$, the right side of (14) converges to 0 . So $a-g_{0}(0)=0$, as wanted.

If $\mu$ has no mass at 1 , then a similar argument, using the left-hand continuity of $g_{0}$ at 1 , shows that $g_{0}(1)=0$. This completes the proof.

Recall that a function $f$ on an interval $U_{0}$ is of bounded mean oscillation on $U_{0}$ if there is a constant $C$ such that $\int_{U}\left|f-f_{U}\right|<C|U|$ for every subinterval $U \subset U_{0}$, where $|U|$ denotes the length of $U$ and $f_{U}$ denotes the average value of $f$ on $U$ (John and Nirenberg [7]). The set of all functions of bounded mean oscillation on $U_{0}$ is a Banach space denoted $B M O\left(U_{0}\right)$, or briefly, BMO.

Proposition 6. Suppose that $L(f)=\int f^{\prime}(x) u(x) d x$ and $G(f)=$ $\int f^{\prime}(x) v(x) d x$ for all $f \in A$, where $u, v \in L^{1}(d x)$. If $G<<<L$ and if $u$ lies in any one of the Banach spaces $B M O$ or $L^{p}(d x), 1<p<\infty$, then $v$ lies in the same Banach space.

Proof. Suppose that $\|G\|_{I^{*}}<K\|L\|_{I^{*}}$ for every ideal $I$.

If $u \in B M O$, then $\int_{U}\left|u-u_{U}\right| d x<C|U|$ for some constant $C$ and all intervals $U=(\alpha, \beta)$. Now if $I=\{f \in A: f(x)=0$ if $x \notin U\}$ then by Proposition 3 we have $\|L\|_{I^{*}}<\int_{U}\left|u-u_{U}\right| d x$. Hence $\|G\|_{I^{*}}<K C|U|$ and by Proposition 3 again we have

$$
\int_{U}\left|v(x)-\int f(t) v(t) d t\right| d x<6 K C|U|
$$

where $f$ is the unit triangle function on $U$. Now it is easy to show that $\int_{U}\left|v-v_{U}\right| d x<2 \int_{U}|v-c| d x$ for any constant $c$ (see John and Nirenberg [7, p. 418]), so that $\int_{U}\left|v-v_{U}\right|<12 K C|U|$. Hence $v \in B M O$, as wanted.

Now suppose that $u \in L^{p}, 1<p<\infty$. We consider $u$ and $v$ as defined on all of $R$ and vanishing off $[0,1]$. We let

$$
(M u)(x)=\sup \left\{|U|^{-1} \int_{U}|u(y)| d y: U \text { an interval, } x \in U\right\}
$$

and

$$
v^{\#}(x)=\sup \left\{|U|^{-1} \int_{U}\left|v(y)-v_{U}\right| d y: U \text { an interval, } x \in U\right\} .
$$


Since $u \in L^{p}$, the maximal function $M u$ is in $L^{p}$, as is well known. Furthermore, to show $v \in L^{p}$, it suffices to show $v^{\#} \in L^{p}$ (Fefferman and Stein [4, Theorem 5, p. 153]). Hence it suffices to show $v^{\#}$ bounded by a constant multiple of $M u$. In fact, we will show

$$
\int_{U}\left|v(y)-v_{U}\right| d y \leqslant 36 K \int_{U}|u(y)| d y
$$

for every interval $U$.

Let $U=(\alpha, \beta)$ be any interval in $\mathbf{R}$. For any function $h$ we will let $h_{U}$ be as above and let $h^{U}=\int_{U} f h d x$ where $f$ is the unit triangle function on $U$. If $0<\alpha<\beta<1$, then

$$
\begin{gathered}
\int_{U}\left|v(y)-v_{U}\right| d y<2 \int_{U}\left|v(y)-v^{U}\right| d y<12\|G\|_{I^{*}} \leqslant 12 K|| L \|_{I^{*}} \\
<12 K \int_{U}\left|u(y)-u^{U}\right| d y<12 K \int_{U}|u(y)| d y+12 K|U|\left|u{ }^{U}\right| \\
<12 K \int_{U}|u|+24 K \int_{U}|u|=36 K \int_{U}|u(y)| d y .
\end{gathered}
$$

If $\alpha<0<\beta$, then

$$
\begin{aligned}
\int_{U^{\prime}}\left|v(y)-v_{U}\right| d y & <2 \int_{U^{\prime}}|v(y)| d y=2 \int_{0}^{\beta}|v(y)| d y<2\|G\|_{I^{*}} \\
& <2 K\|L\|_{I^{*}}<2 K \int_{0}^{\beta}|u(y)| d y=2 K \int_{U^{\prime}}|u(y)| d y .
\end{aligned}
$$

The case $\alpha<1<\beta$ is similar to the case just done. Finally, if $U$ does not intersect $[0,1]$ at all, then both sides of (15) are 0 . Thus (15) and the proposition have been proven.

Proposition 7. Suppose that $L(f)=a f(0)+\int f^{\prime} d \mu$ and $G(f)=b f(0)+$ $\int f^{\prime} d \nu$ for all $f \in A$. If $G<<<L$, and if there exist $r \in(0,1)$ and a constant $C$ such that $|\mu|(U)<C|U|^{r}$ for every closed interval $U \in[0,1]$, then there exists a constant $D$ such that $|\nu|(U)<D|U|^{r}$ for every such $U$.

Proof. Suppose $\|G\|_{I^{*}}<K\|L\|_{I^{*}}$ for all closed ideals $I$. Clearly $\mu$ has no point masses, so that $\nu$ has none either. Suppose $0<\alpha<\beta<1$ and let $I=\{f \in A: f(x)=0$ if $x \notin(\alpha, \beta)\}$. Then $\|L\|_{I^{*}}<|\mu|(\alpha, \beta)<C(\beta-\alpha)^{r}$. Choosing $f_{n}$ as in the proof of Proposition 5

$$
\left|\int f_{n}^{\prime} d \nu\right|<2\|G\|_{I^{*}}<2 C K(\beta-\alpha)^{r} .
$$

Letting $n \rightarrow \infty$, we get $|\nu(\alpha, m)-\nu(m, \beta)|<2 C K(\beta-\alpha)^{r}$, where $m=(\alpha$ $+\beta) / 2$. If we define $F(x)=\nu(0, x)$ and $h=(\beta-\alpha) / 2$, then $F(m+h)-$ $2 F(m)+F(m-h)=\nu(m, \beta)-\nu(\alpha, m)$, so that 


$$
|F(m+h)-2 F(m)+F(m-h)|<2 C K 2^{r} h^{r}
$$

whenever $0 \leqslant m-h<m+h<1$. But this implies that $F$ satisfies a Lipschitz condition of order $r$ (Stein [10, Proposition 8, p. 146]), that is,

$$
|F(m+h)-F(m)| \leqslant D h^{r}
$$

for some constant $D$ and all $m, h$ such that $0 \leqslant m<m+h \leqslant 1$. Hence $|\nu|(m, m+h)<D h^{r}$, as wanted.

In view of the last few results it is interesting to consider the map $L \mapsto \Phi L$ of $A^{*}$ into itself, for a fixed $\Phi \in A^{* *}$. We consider $\mathbf{C} \oplus L^{1}(d x)$ as a subspace of $A^{*}$; the pair $(a, u) \in \mathbf{C} \oplus L^{1}(d x)$ determines the linear functional $L$ given by $L(f)=a f(0)+\int f^{\prime} u d x$. If $\Phi \in A^{* *}$, then the restriction of $\Phi$ to $\mathbf{C} \oplus$ $L^{1}(d x)$ determines a pair $(b, \theta) \in \mathbf{C} \oplus L^{\infty}(d x)$ such that $\Phi(L)=a b+$ $\int u(x) \theta(x) d x$. In the proof of Proposition 4 we calculated $g L$ for $g \in A$. Using this, a tedious calculation shows

$$
\begin{aligned}
(\Phi L)(f)= & f(0)\left(a b+\int u \theta d x\right) \\
& +\int_{0}^{1} f^{\prime}(t)\left[\left(b+\int_{0}^{t} \theta(x) d x\right) u(t)+\int_{t}^{1} \theta(x) u(x) d x\right] d t .
\end{aligned}
$$

Hence the mapping of $L^{1}(d x)$ into itself that sends $u$ into the function $\left(b+\int_{0}^{t} \theta(x) d x\right) u(t)+\int_{t}^{1} \theta(x) u(x) d x$, for fixed $(b, \theta) \in \mathbf{C} \oplus L^{\infty}(d x)$, is "oscillation decreasing." Note that if $u$ is sufficiently smooth, then the derivative of the latter function is $\left(b+\int_{0}^{t} \theta(x) d x\right) u^{\prime}(t)$, so that we can think of this mapping as differentiation, followed by multiplication by an absolutely continuous function, followed by integration.

Finally we wish to mention that Arens multiplication is commutative, as can be shown by the same type of calculation used to establish (16).

EXAMPLE 9. Let $A$ be the algebra of continuous functions on the circle $T=\{z:|z|=1\}$ whose negative Fourier coefficients vanish, that is, $A=\{f$ $\in C(T): \int e^{i n t} f(t) d t=0$ if $\left.n>0\right\}$. Each $f \in A$ can be extended uniquely to a continuous function on the disc $\{z:|z|<1\}$ which is holomorphic in the interior $\{z:|z|<1\}$ and we shall identify $f$ with this extension.

Now $A$ is a subalgebra of $C(T)$ and its dual is $M(T) / H_{0}^{1}$, where $M(T)$ is the set of regular Borel measures on $T$ and $H_{0}^{1}$ is the subset of measures which annihilate $A$, that is, $H_{0}^{1}=\left\{\mu: \int_{0}^{2 \pi} e^{i n t} d \mu(t)=0\right.$ if $\left.n \geqslant 0\right\}$. By the theorem of F. and M. Riesz, all measures in $H_{0}^{1}$ are absolutely continuous with respect to Lebesgue measure.

The ideals in $A$ can be described as follows (Hoffman [6, p. 84]): Let $K$ be a subset of $T$ of Lebesgue measure 0 and let $F$ be an inner function such that

1. if $\alpha_{1}, \alpha_{2}, \ldots$ are the zeroes of $F$ in the interior of the unit disc, then every accumulation point of the $\alpha_{n}$ is in $K$, and 
2. the measure determining the singular part of $F$ is supported on $K$.

Then $I=I(K, F)=\{F f: f \in A$ and $f(x)=0$ if $x \in K\}$ is an ideal in $A$. Conversely, every ideal $I$ in $A$ is of this form.

Proposition 8. Let $K, F$, and $I=I(K, F)$ be as above. Let $\mu$ be a measure on $T$ and let $L(f)=\int f d \mu$ for $f \in A$. Then

$$
\|L\|_{I^{*}} \leqslant\left\|\left.F d \mu\right|_{\sim K}\right\|_{A^{*}}<2\|L\|_{I^{*}}
$$

where $\left.F d \mu\right|_{\sim K}$ denotes the restriction of the measure $F d \mu$ to the complement of K.

Proof. Note that $F$ is continuous off $K$ so that $\left.F d \mu\right|_{\sim K}$ is well-defined.

By Fatou's theorem (Hoffman [6, p. 80]) we can choose a function $h \in A$ such that $h(x)=1$ if $x \in K$ and $|h(x)|<1$ if $x \notin K$. Then for any $f \in A$ and any positive integer $n$ we have $\left(f-f h^{n}\right) F \in I$ and $\left\|\left(f-f h^{n}\right) F\right\|<$ $2\|f\|$. Hence

$$
\left|\int\left(f-f h^{n}\right) F d \mu\right| \leqslant\left\|\left(f-f h^{n}\right) F\right\|\|L\|_{I^{*}} \leqslant 2\|F\|\|L\|_{I^{*}}
$$

Letting $n \rightarrow \infty$ gives $\left|\int_{\sim K} f F d \mu\right| \leqslant 2\|f\|\|L\|_{I^{*}}$. This proves the second half of (17).

On the other hand, if $F f \in I$, then $|L(F f)|=\left|\int F f d \mu\right|=\left|\int_{\sim K} f F d \mu\right|<$ $\|f\|\left\|\left.F d \mu\right|_{\sim K}\right\|_{A^{*}}$. This proves the first half of (17).

If $\mu \in M=M(T)$ then $\|\mu\|_{A^{*}}=\inf \left\{\|\mu+h\|_{M}: h \in H_{0}^{1}\right\}$. We will need the following simple lemma.

LEMMA. If $u \in L^{1}(d x)$ and do is a singular measure, then

(a) $\|u d x+d \sigma\|_{A^{*}}=\|u d x\|_{A^{*}}+\|d \sigma\|_{A^{*}}$, and

(b) $\|d \sigma\|_{A^{*}}=\|d \sigma\|_{M^{*}}$

PRoof. (a) If $h \in H_{0}^{1}$ then $\|u d x+d \sigma+h d x\|_{M}=\|u d x+h d x\|_{M}+$ $\|d \sigma\|_{M} \geqslant\|u d x\|_{A^{*}}+\|d \sigma\|_{A^{*}}$, and taking the infimum of the left hand side over all such $h$ gives the result.

(b) If $h \in H_{0}^{1}$ then $\|d \sigma+h d x\|_{M}=\|d \sigma\|_{M}+\|h d x\|_{M}>\|d \sigma\|_{M}$, so that $\|d \sigma\|_{A^{*}} \geqslant\|d \sigma\|_{M}$. The reverse inequality is clear.

Recall that a function on $T$ is said to be of bounded characteristic if it is the quotient of two functions in $H^{1}$. If a function $u$ in $L^{1}(d x)$ is of bounded characteristic, then $u$ has a unique factorization of the form $u=H / F$ where $F$ is an inner function and $H$ is a function in $H^{1}$ such that $F$ and the inner part of $H$ have no common divisor. Integrable functions of bounded characteristic are dense in $L^{1}(d x)$, for otherwise there would be a nonzero function in $L^{\infty}(d x)$ which annihilates $z^{n}$ for all integers $n$, an impossibility.

We can now describe the notion of absolute continuity in $A$.

Proposition 9. Let $L(f)=\int f d \mu$ and $G(f)=\int f d \nu$ for all $f \in A$, where 
$d \mu=u d x+d \sigma, d \nu=v d x+d \tau, u$ and $v$ are in $L^{1}(d x)$, and $\sigma$ and $\tau$ are singular measures. Then $G \ll L$ if and only if $G$ is in the closure of $\{g L$ : $g \in A\}$. Furthermore:

(a) Suppose that $u$ is of bounded characteristic with factorization $u=H / F$. Then $G \ll L$ if and only if each of the following three conditions is true:

(i) $F v \in H^{1}$,

(ii) if the meromorphic extension of $u$ to the disc has a zero at $z=0$ then the meromorphic extension of $v$ to the disc (which exists by (i)) also has a zero at $z=0$,

(iii) $\tau$ is absolutely continuous with respect to $\sigma$.

(b) Suppose that $u$ is not of bounded characteristic. Then $G \ll L$ if and only if $v \in L^{1}(d x)$ and $\tau$ is absolutely continuous with respect to $\sigma$.

Proof. (a) Suppose that conditions (i), (ii), (iii) are satisfied. We will show $G \ll L$ by showing that $G$ is in the closure of $\{g L: g \in A\}$. Note that $(g L)(f)=\int f(g u d x+g d \sigma)$. If $G$ is not in the closure of $\{g L: g \in A\}$ then by the Hahn-Banach Theorem we can find a linear functional annihilating $\{g L: g \in A\}$ but not $G$. This linear functional on $A^{*}=M(T) / H_{0}^{1}$ can be restricted to $L^{1}(d x)+L^{1}(d \sigma) / H_{0}^{1}$ to give a pair $\left(\theta_{1}, \theta_{2}\right) \in L^{\infty}(d x)+L^{\infty}(d \sigma)$ which annihilates $H_{0}^{1}$, where the action of $\left(\theta_{1}, \theta_{2}\right)$ on $\left(f_{1}, f_{2}\right) \in L^{1}(d x)+$ $L^{1}(d \sigma)$ is given by $\left(\theta_{1}, \theta_{2}\right)\left(f_{1}, f_{2}\right)=\int f_{1} \theta_{1} d x+\int f_{2} \theta_{2} d \sigma$. The condition that $\left(\theta_{1}, \theta_{2}\right)$ annihilates $H_{0}^{1}$ and $\{g L: g \in A\}$ becomes

$$
\int \theta_{1} h d x=0 \text { if } h \in H_{0}^{1} \text { and } \int\left(\theta_{1} g u d x+\theta_{2} g d \sigma\right)=0 \text { if } g \in A \text {. }
$$

The first equation says $\theta_{1} \in H^{\infty}$ and the second says that $\theta_{1} u d x+\theta_{2} d \sigma \in$ $H_{0}^{1}$. By the theorem of F. and M. Riesz, we have $\theta_{2} d \sigma=0$ and $\theta_{1} u \in H_{0}^{1}$. But $\theta_{1} u=\theta_{1} H / F$, so that $\theta_{1} / F$ is holomorphic (and bounded) in the disc and either $H$ or $\theta_{1} / F$ has a zero at the origin. Therefore, $v \theta_{1}=v F\left(\theta_{1} / F\right) \in$ $H^{1}$ by (i). Furthermore, if $\theta_{1} / F$ has a zero at the origin, then so does $v \theta_{1}$, so that $v \theta_{1} \in H_{0}^{1}$; on the other hand, if $\theta_{1} / F$ does not have a zero at the origin, then $H$ has a zero at the origin, hence $u$ has a zero at $z=0$, hence by (ii) $v$ has a zero at $z=0$, hence $v \theta_{1} \in H_{0}^{1}$. Therefore, in any case, $\int\left(v \theta_{1} d x+\right.$ $\left.\theta_{2} d \tau\right)=0$, that is, $\left(\theta_{1}, \theta_{2}\right)$ annihilates $G$, contrary to the assumption about $\left(\theta_{1}, \theta_{2}\right)$. This proves that $G$ is in the closure of $\{g L: g \in A\}$, as wanted.

Conversely, suppose that $G \ll L$. Decompose $\tau, \tau=\tau_{1}+\tau_{2}$, where $\tau_{1}$ is absolutely continuous with respect to $\sigma$ and $\tau_{2}$ is singular with respect to $\sigma$. Now the inner function $F$ is the uniform limit of Blaschke products (Hoffman [6, p. 175]) and a Blaschke product is the limit a.e. of finite Blaschke products (Hoffman [6, p. 65]), so that we can find a sequence $F_{n}$ of finite Blaschke products such that $F_{n} \rightarrow F$ a.e. We can also find an increasing sequence $K_{n}$ of compact sets of Lebesgue measure 0 such that $\left|\tau_{2}\right|\left(K_{n}\right)=0$ and $d \sigma$ is supported on the union of the $K_{n}$. Let $I_{n}=I_{n}\left(K_{n}, F_{n}\right)$ be the ideal in $A$ determined 
by $K_{n}$ and $F_{n}$. Then by the preceding proposition and lemma,

$$
\|L\|_{I_{n}^{*}} \leqslant 2\left\|\left.F_{n}(u d x+d \sigma)\right|_{\sim K_{n}}\right\|_{A^{*}}=2\left\|F_{n} u\right\|_{A^{*}}+2\left\|\left.F_{n} d \sigma\right|_{\sim K_{n}}\right\|_{M}
$$

But $\left\|F_{n} u\right\|_{A^{*}}<\left\|F_{n} u-H\right\|_{M}=\left\|F_{n} H-F H\right\|_{L^{1}} \rightarrow 0$ by the Lebesgue Dominated Convergence Theorem and $\left\|\left.F_{n} d \sigma\right|_{\sim K_{n}}\right\| \rightarrow 0$ by the definition of $K_{n}$. Hence $\|G\|_{I_{n}^{*} \rightarrow 0 \text {. But }}$

$$
\begin{aligned}
\|G\|_{I_{n}^{*}} & \geqslant\left\|\left.F_{n}\left(v d x+d \tau_{1}+d \tau_{2}\right)\right|_{\sim K_{n}}\right\|_{A^{*}} \\
& =\left\|F_{n} v\right\|_{A^{*}}+\left\|\left.F_{n} d \tau_{1}\right|_{\sim K_{n}}\right\|+\left\|\left.F_{n} d \tau_{2}\right|_{\sim K_{n}}\right\|
\end{aligned}
$$

from which it follows that each of the three terms on the right hand side goes to 0 as $n \rightarrow \infty$. Now the Lebesgue Dominated Convergence Theorem implies $F_{n} v \rightarrow F v$ in $L^{1}$, and since $\left\|F_{n} v\right\|_{A^{*}} \rightarrow 0$ we have $F v \in H_{0}^{1}$, proving (i). Furthermore, if $u$ is 0 at $z=0$, then $F$ is not 0 at $z=0$, so that $v$ must have a zero at the origin, proving (ii). Finally, note that $\left\|\left.F_{n} d \tau_{2}\right|_{\sim K_{n}}\right\|_{A^{*}}=\left\|F_{n} d \tau_{2}\right\|_{M}$ $=\left\|d \tau_{2}\right\|_{M}$, since $\left|F_{n}\right|$ is 1 on the circle, so that $\tau_{2}=0$ and $\tau$ is absolutely continuous with respect to $\sigma$, proving (iii).

(b) First we prove that if $\tau$ is absolutely continuous with respect to $\sigma$ and $v \in L^{1}(d x)$ then $G$ is in the closure of $\{g L: g \in A\}$, hence $G \ll L$. For if not, then proceeding as in part (a) we find a pair $\left(\theta_{1}, \theta_{2}\right) \in L^{\infty}(d x)+L^{\infty}(d \sigma)$ which annihilates $H_{0}^{1}$ and $\{g L: g \in A\}$ but not $G$. As in (a) we conclude $\theta_{1} \in H, \theta_{2} d \sigma=0$, and $\theta_{1} u \in H_{0}^{1}$. If $\theta_{1}$ is not identically 0 then $u=\left(\theta_{1} u\right) / \theta_{1}$ is of bounded characteristic, contrary to our assumption. Hence $\theta_{1}=0$ and $\left(\theta_{1}, \theta_{2}\right)$ annihilates $G$. This contradiction proves that $G$ is in the closure of $\{g L: g \in A\}$.

Conversely, suppose $G \ll L$. Decompose $\tau=\tau_{1}+\tau_{2}$ as in (a). Since integrable functions of bounded characteristic are dense in $L^{1}(d x)$, for any $n$ we can find $H_{n} \in H^{1}$ and an inner function $F_{n}$ such that $\left\|u-H_{n} / F_{n}\right\| \rightarrow 0$ as $n \rightarrow \infty$. Furthermore, we can assume as in (a) that $F_{n}$ is a finite Blaschke product, and we can obtain a compact set $K_{n}$ and an ideal $I_{n}$ as in (a) such that (18) holds. But $\left\|F_{n} u\right\|_{A^{*}} \leqslant\left\|F_{n} u-H_{n}\right\|_{L^{1}}=\left\|u-H_{n} / F_{n}\right\|_{L^{1}} \rightarrow 0$ and $\left.F_{n} d \sigma\right|_{\sim K_{n}} \rightarrow 0$. Hence $\|G\|_{I_{n}^{*}} \rightarrow 0$ and as in (a) we find that $\tau_{2}=0$, as wanted.

3. Generalizations. Let $A$ be a Banach algebra, not necessarily commutative, and let $B$ be a Banach module over $A$, that is, $B$ is a Banach space, and there is a representation of $A$ on $B$ such that $\|a f\|_{B} \leqslant\|a\|_{A}\|f\|_{B}$ for all $a \in A$ and $f \in B$. If $L, G \in B^{*}$, then we define $G \ll L$ to mean that for every $\varepsilon>0$ there exists $\delta>0$ such that if $\|L\|_{I^{*}} \leqslant \delta$, where $I$ is a closed invariant subspace of $B$, then $\|G\|_{I^{*}} \leqslant \varepsilon$. We define $G<<<L$ to mean that $\|G\|_{I^{*}} \leqslant K\|L\|_{I^{*}}$, for some constant $K$ and all invariant subspaces $I \subset B$.

For example, suppose that we have a representation of $A$ on a Hilbert space $H$. If the representation is irreducible, so that the only invariant 
subspaces are 0 and $H$, then $G \ll L$ whenever $G, L \in H^{*}=H$ and $L \neq 0$. Suppose more generally $H=\bigoplus_{\alpha} H_{\alpha}$, where each $H_{\alpha}$ is irreducible, and $L(f)=\langle f, \mu\rangle$ for some $\mu \in H$ and all $f \in H$, where $\langle\cdot, \cdot\rangle$ is the inner product in $H$. If $\mu=\Sigma \mu_{\alpha}, \mu_{\alpha} \in H_{\alpha}$, then $\|L\|_{H_{\alpha}^{*}}=\left\|\mu_{\alpha}\right\|$. Hence if $G<<<$ $L$, where $L(f)=\langle f, \mu\rangle$ and $G(f)=\langle f, \nu\rangle$, then $\left\|\nu_{\alpha}\right\|\left\langle K\left\|\mu_{\alpha}\right\|\right.$ for all $\alpha$ and some constant $K$. The converse is easy to prove. Also, if merely $G \ll L$, then $\nu_{\alpha}=0$ if $\mu_{\alpha}=0$, and this converse is also easy to prove.

Now suppose again that $B$ is a Banach module over $A$. If $a \in A$ and $L \in B^{*}$, we define $a L$ on $B$ by $(a L)(f)=L(a f)$ for $f \in B$. It is clear that $a L \in B^{*}$ and $\|a L\|_{I^{*}} \leqslant\|a\|\|L\|_{I^{*}}$ for all invariant $I$ in $B$. Thus $a L<<<L$. A Radon-Nikodym theorem for $B$ would say that if $G \ll L$ then $G$ is in the norm closure of $\{a L: a \in A\}$. We will content ourselves with examining several examples.

EXAMPLE 10. Let $G$ be a compact group with normalized Haar measure and let $A=L^{2}(G)$. With the convolution

$$
f * g(y)=\int_{G} f\left(y x^{-1}\right) g(x) d x
$$

$A$ becomes a Banach algebra, and we let $A$ act on itself by left multiplication.

If $R$ is an irreducible representation of $G$ on a $d$-dimensional Hilbert space $H$, choose an orthogonal basis $v_{1}, \ldots, v_{d}$ of $H$ and let $\phi_{i j}(x)=\left\langle R_{x} v_{i}, v_{j}\right\rangle$. For each $j$, the linear span of $\left\{\phi_{i j}: i=1, \ldots, d\right\}$ is a left invariant subspace of $A$. By the Peter-Weyl theorem, $L^{2}(G)=\bigoplus H_{\alpha}$, where each $H_{\alpha}$ is the span of the $\left\{\phi_{i j}\right\}$ determined by an irreducible representation.

If $\mu \in A^{*}$, we can identify $\mu$ with a function (also denoted $\mu$ ) in $L^{2}(G)$ such that $\mu(f)=\int f(x) \overline{\mu(x)} d x$. A straightforward calculation shows that $f \mu=$ $f^{*} * \mu$ for all $f \in A, \mu \in A^{*}$, where $f^{*}(x)=\overline{f\left(x^{-1}\right)}$. The Radon-Nikodym theorem for $A$ would say that if $\nu \ll \mu$, then $f \in A$ can be chosen so that $f * \mu$ is arbitrarily close to $\nu$. Now if $f=\Sigma f_{\alpha}, \mu=\Sigma \mu_{\alpha}, \nu=\Sigma \nu_{\alpha}$, then $f * \mu=$ $\Sigma\left(f_{\alpha} * \mu_{\alpha}\right)$, so it is sufficient to find $f_{\alpha}$ so that $f_{\alpha} * \mu_{\alpha}$ is near $\nu_{\alpha}$, for each $\alpha$ (that is, for each irreducible representation). Now suppose that $f_{\alpha}=\Sigma f_{i j} \phi_{i j}$, $\mu_{\alpha}=\sum \mu_{i j} \phi_{i j}, \nu_{\alpha}=\sum \nu_{i j} \phi_{i j}$. Then $f_{\alpha} * \mu_{\alpha}=d^{-1} \Sigma_{i, l}\left(\Sigma_{j} f_{i j} \mu_{j l}\right) \phi_{i l}$, where $d$ is the dimension of the representation. Letting $\left[q_{i j}\right]$ denote the matrix which has $q_{i j}$ in the $i$ th row and $j$ th column, we see that we want $\left[f_{i j}\right]$ so that the components of $\left[f_{i j}\right]\left[\mu_{i j}\right]$ are close to those of $\left[\nu_{i j}\right]$. Now the assumption $\nu \ll \mu$ implies that if a column $\left\{\mu_{i j}: i=1, \ldots, d\right\}$ is 0 then the corresponding $\nu$-column is 0 , which is clearly necessary for the existence of $f$. However, it is not sufficient. For, if two columns of $\left[\mu_{i j}\right]$ are identical but not 0 and the corresponding two columns of $\left[\nu_{i j}\right]$ are different, then we cannot find $\left[f_{i j}\right]$ making $\left[f_{i j}\right]\left[\mu_{i j}\right]$ arbitrarily close to $\left[\nu_{i j}\right]$. Hence the Radon-Nikodym theorem need not be true. If, however, the nonzero columns of $\left[\mu_{i j}\right]$ are linearly independent, then $\left[f_{i j}\right]$ can be found so that $\left[f_{i j}\right]\left[\mu_{i j}\right]=\left[\nu_{i j}\right]$. For example, if $\mu$ 
is a central function (that is, if $\mu(x y)=\mu(y x)$ for all $x, y \in G$ ) then $\mu_{i j}=0$ if $i \neq j$, making the nonzero columns independent, and the Radon-Nikodym theorem holds for central functions.

EXAMPLE 11. Let $H$ be a separable infinite dimensional Hilbert space and let $\mathrm{C}(H)$ be the Banach algebra of compact operators on $H$. The dual space of $\mathcal{C}(H)$ can be identified with the Banach space $\mathcal{T}(H)$ of trace class operators on $H$ with the trace norm $\|\cdot\|_{1}$. The duality is given by the bilinear form $\operatorname{tr}(T C)=\operatorname{trace}(T C), T \in \mathcal{T}(H), C \in \mathcal{C}(H)$.

Suppose that $T$ is a trace class operator on $H$ and that $\left\{x_{j}: j=\right.$ $1,2,3, \ldots\}$ is an orthonormal basis in $H$. Fix any integer $n$ and let $I=\left\{C \in \mathcal{C}(H): C\left(x_{j}\right)=0\right.$ if $\left.j \neq n\right\}$, which is clearly a closed left ideal in $\mathcal{C}(H)$. If $E$ is the projection on the span of $x_{n}$, then $\left.\|T\|_{I^{*}}\right\rangle|\operatorname{tr}(T E)|=\left|\Sigma_{j}\left\langle T E x_{j}, x_{j}\right\rangle\right|=\left|\left\langle T x_{n}, x_{n}\right\rangle\right|$. That is,

$$
\|T\|_{I^{*}} \geqslant\left|\left\langle T\left(x_{n}\right), x_{n}\right\rangle\right| \quad(T \in \mathcal{T}(H)) .
$$

If in addition $x_{n}$ is an eigenvector of $\bar{T}$ (the adjoint of $T$ ) then for every $C \in I$ we have

$$
\begin{aligned}
|\operatorname{tr}(T C)| & =\left|\sum_{j}\left\langle T C x_{j}, x_{j}\right\rangle\right|=\left|\left\langle C x_{n}, \bar{T} x_{n}\right\rangle\right| \\
& =\left|\left\langle T x_{n}, x_{n}\right\rangle\left\langle C x_{n}, x_{n}\right\rangle\right| \leqslant\left|\left\langle T x_{n}, x_{n}\right\rangle\right|\|C\|,
\end{aligned}
$$

so that

$$
\|T\|_{I^{*}}=\left|\left\langle T x_{n}, x_{n}\right\rangle\right| \quad\left(T \in \mathcal{T}(H), x_{n} \text { eigenvector of } \bar{T}\right) .
$$

Now suppose that $A, B \in \mathcal{T}(H)$, with $A$ normal, and that $B<<<A$. If $\left\{x_{n}\right\}$ is an orthonormal basis of eigenvectors of $A$ and if $\|B\|_{I^{*}}<K\|A\|_{I^{*}}$ for all $I$, then we have

$$
\left|\left\langle B x_{n}, x_{n}\right\rangle\right| \leqslant\|B\|_{I^{*}} \leqslant K\|A\|_{I^{*}}=K\left|\left\langle A x_{n}, x_{n}\right\rangle\right|=K\left\langle|A| x_{n}, x_{n}\right\rangle .
$$

Now this does not in general imply that $|B| \leqslant K|A|$. However, if the $x_{n}$ can be chosen so that they are eigenvectors of $B$ as well (that is, if $A$ and $B$ can be simultaneously diagonalized), then we have $\left\langle(K|A|-|B|) x_{n}, x_{n}\right\rangle \geqslant 0$ for all $n$ and since the $x_{n}$ are eigenvectors of $K|A|-|B|$, we have $|B|<K|A|$. If $A x_{n}=\mu_{n} x_{n}$ and $B x_{n}=\nu_{n} x_{n}$, then $\left|\nu_{n}\right|<K\left|\mu_{n}\right|$ for all $n$. If $D_{n}\left(x_{j}\right)=\nu_{j} / \mu_{j}$ for $j=1, \ldots, n$ and $D_{n}\left(x_{j}\right)=0$ if $j>n$, then the $D_{n}$ are uniformly bounded compact operators and $B=\lim D_{n} A$. Note that if we had merely assumed $B \ll A$, then $\nu_{n}=0$ whenever $\mu_{n}=0$, so that $D_{n}$ can still be found so that $B=\lim D_{n} A$, although the $D_{n}$ are not necessarily uniformly bounded. In any case, a Radon-Nikodym theorem holds only when additional commutativity conditions are placed upon the "linear functionals" $A$ and $B$.

For a different approach, we recall the Radon-Nikodym theorems of Sakai for $W^{*}$-algebras. Suppose that $\mathscr{B}$ is a $W^{*}$-algebra and let $\phi, \psi$ be 
positive normal linear functionals on $\mathscr{B}$ such that $\psi<\phi$. Then there exist positive elements $t$ and $h$ in $\mathscr{B}$ such that $0<t<1,0<h<1$, and $\psi(f)=\phi(t f t)=\frac{1}{2} \phi(h f+f h)$ for all $f \in \mathscr{B}$ (Sakai [9, pp. 76-77]). If $\mathscr{B}=$ $\mathscr{B}(H)$, then the normal linear functionals on $\mathscr{B}$ are the trace class operators (that is, $\mathscr{B}$ is the dual of $\mathscr{T}(H)$ ), and it is interesting to note that if we change our definition of absolute continuity slightly, the relation $<<<$ in $\mathscr{T}(H)$ (considered as the dual of $\mathcal{C}(H)$ ) becomes almost identical to the relation < in $\mathscr{T}(H)$ (considered as the predual of $\mathscr{B}(H)$ ), which is the hypothesis of Sakai's Radon-Nikodym theorems. The change consists of considering norms of restrictions to $I \cap \bar{I}$ rather than $I$, where $I$ is a closed left ideal and $\bar{I}=\{\bar{A}: A \in I\}(\bar{A}=$ adjoint of $A)$.

Proposition 10. Let $A$ and $B$ be positive trace class operators on $H$. Then $B<A$ if and only if

$$
\|B\|_{(I \cap \bar{I})^{*}} \leqslant\|A\|_{(I \cap \bar{I})^{*}}
$$

for every closed left ideal $\mathrm{I}$ in $\mathrm{C}(H)$.

Proof. Note that if $E$ is an orthogonal projection, then $I=\{C \in \mathcal{C}(H)$ : $C E=0\}$ is a closed left ideal. Furthermore every closed left ideal in $\mathcal{C}(H)$ has this form (Rickart [8, p. 284]). Given $E$ and $I$, let $F=1-E$ and note that $(I \cap \bar{I})^{*}=\{C \in \mathcal{C}(H): C E=E C=0\}$. If $A \in \mathcal{T}(H)$ and $A>0$, we claim that $\|A\|_{(I \cap \bar{I})^{*}}=\operatorname{tr}(F A F)$. For, if $C \in(I \cap \bar{I})^{*}$, then $|\operatorname{tr}(A C)|=$ $|\operatorname{tr}(A F C F)|=|\operatorname{tr}(F A F C)|<\operatorname{tr}(F A F)\|C\|$, so that $\|A\|_{(I \cap \bar{I})^{*}}<\operatorname{tr}(F A F)$. But if $\left\{x_{j}: j=1,2,3, \ldots\right\}$ is an orthogonal basis for the range of $F$ and if $C_{n}$ is the projection on the span of $\left\{x_{1}, x_{2}, \ldots, x_{n}\right\}$, then

$$
\|A\|_{(I \cap \bar{I})^{*}}>\operatorname{tr}\left(A C_{n}\right)=\left|\sum_{j=1}^{n}\left\langle A x_{j}, x_{j}\right\rangle\right|=\left|\sum_{j=1}^{n}\left\langle F A F x_{j}, x_{j}\right\rangle\right|
$$

and letting $n \rightarrow \infty$ gives $\|A\|_{(I \cap \bar{I})^{*}} \geq \operatorname{tr}(F A F)$.

Now if $0 \leqslant B<A, A, B \in \mathcal{T}(H)$, and $I=\{C: C E=0\}$, then $F B F<$ $F A F$ and $\|B\|_{\left(I \cap \bar{I}^{*}\right.}=\operatorname{tr}(F B F) \leqslant \operatorname{tr}(F A F)=\|A\|_{(I \cap \bar{I})^{* *}}$ Conversely, if (19) holds and $x \in H$, let $F$ be the orthogonal projection on the span of $x$ and let $E=1-F$. Then

$$
\langle B x, x\rangle=\operatorname{tr}(F B F)=\|B\|_{(I \cap \bar{I})^{*}}\left\langle\|A\|_{(I \cap \bar{I})^{*}}=\operatorname{tr}(F A F)=\langle A x, x\rangle,\right.
$$

so that $B<A$.

\section{BIBLIOGRAPHY}

1. R. Arens, Operations induced in function classes, Monatsh. Math. Physik 55 (1951), 1-19.

2. __ The adjoint of a bilinear operation, Proc. Amer. Math. Soc. 2 (1951), 839-848.

3. P. Civin and B. Yood, The second conjugate of a Banach algebra as an algebra, Pacific J. Math. 11 (1961), 847-870. 
4. C. Fefferman and E. M. Stein, $H^{p}$ spaces of several variables, Acta Math. 129 (1972), 137-193.

5. E. Hewitt and K. Ross, Abstract harmonic analysis. II, Springer-Verlag, Berlin and New York, 1970. 1962.

6. K. Hoffman, Banach spaces of analytic functions, Prentice-Hall, Englewood Cliffs, N. J.,

7. F. John and L. Nirenberg, On functions of bounded mean ascillation, Comm. Pure Appl. Math. 14 (1961), 415-426.

8. C. E. Rickart, General theory of Banach algebras, Van Nostrand, Princeton, N. J., 1960.

9. S. Sakai, $C^{*}$-algebras and $W^{*}$-algebras, Springer-Verlag, Berlin and New York, 1970.

10. E. M. Stein, Singular integrals and differentiability properties of functions, Princeton Univ. Press, Princeton, N. J., 1970.

Singer-Link, 1077 East Arques Avenue, Sunnyvale, California 94086 